\title{
A Comparative Study of Overtone CH-Stretching Vibrational Circular Dichroism Spectra of Fenchone and Camphor ${ }^{\dagger}$
}

\author{
Giovanna Longhi,,$\S$ Roberto Gangemi,,$\$$ France Lebon,,,$\S$ Ettore Castiglioni, \\ Sergio Abbate, $*,, \S$ Vaughan M. Pultz, and David A. Lightner ${ }^{\perp}$ \\ Dipartimento di Scienze Biomediche e Biotecnologie, Università di Brescia, viale Europa 11, \\ 25123 Brescia, Italy, INFM-Istituto Nazionale di Fisica della Materia, UdR Brescia via Valotti 9, \\ 25123 Brescia, Italy, Science Division, Truman State University, Kirksville, Missouri 63501, and \\ Department of Chemistry, University of Nevada, Reno, Nevada 89557
}

Received: February 9, 2004; In Final Form: March 30, 2004

\begin{abstract}
Near-infrared vibrational circular dichroism (VCD) spectra in the region 1500-850 nm are recorded for both enantiomers of camphor and fenchone using a home-built dispersive instrument. Two $\mathrm{CH}$-stretching overtone regions and two $\mathrm{CH}$-stretching/ $\mathrm{HCH}$-bending combination regions are investigated. The near-infrared VCD data are examined and compared with the corresponding previously published data of $(3 R)-(+)$-methylcyclopentanone and $(3 R)-(+)$-methylcyclohexanone. The absorption spectra of both the fundamental and overtone regions are also considered, and the fundamental $\mathrm{CH}$-stretching VCD data are taken into account, where possible. An empirical assignment of the spectra is first considered, with special regard to the second $\mathrm{CH}-$ stretching overtone region. This region is then investigated by a heuristic approach based on DFT calculations, using the hybrid functional B3LYP at the 6-31G** basis set level. Subsequently rotational strengths have been evaluated by use of a classical model based on the contribution of polarizable groups to the optical activity. The same models are also used to interpret the VCD spectra between 2000 and 3000 wavenumbers for $(3 R)-(+)$-methylcyclopentanone- $d_{4}$.
\end{abstract}

\section{Introduction}

Camphor and fenchone are natural products belonging to the class of essential oils. They are optically active, and the most abundant enantiomers are (+)-camphor, which is extracted mainly from the bark of the tree cinnamonum camphora, and (-)-fenchone, which is abundant in fennel. They are useful as test compounds for basic chemical studies, especially in relation to their chiroptical properties. Indeed the two molecules $\mathbf{1}$ and $\mathbf{2}$, for which the structures of the $(1 S)$-enantiomers are reported below, were investigated by circular dichroism (CD) spectroscopy, both in the near-UV ${ }^{1,2}$ and in the vacuum UV region, ${ }^{3,4}$ by vibrational circular dichroism (VCD) spectroscopy and through vibrational analysis. ${ }^{5-9}$

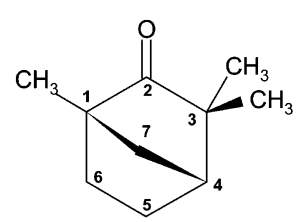

(1S)-(+)- fenchone

(1)

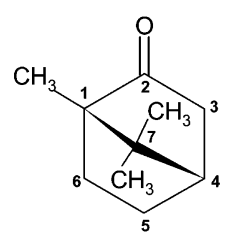

(1S)-(-) camphor

(2)
Recently the investigation of the specific rotation by means of current theory has also been closely considered, and matching theory with experiment would be quite desirable and informa-

\footnotetext{
$\dagger$ Work presented at the CD2003 Conference in Budapest, Hungary, September 4, 2003.

* To whom correspondence should be addressed. E-mail: abbate@ med.unibs.it.

† Università di Brescia.

$\S$ INFM-Istituto Nazionale di Fisica della Materia.

" Truman State University.

$\perp$ University of Nevada.
}

tive. ${ }^{10-12}$ Both types of molecules are structurally quite rigid, and this constitutes an important reason for studying them, even though they may look rather complex because of the high number of vibrational degrees of freedom.

In this work we consider the VCD spectra of $\mathbf{1}$ and $\mathbf{2}$ and of their enantiomeric counterparts in the $\mathrm{CH}$-stretching overtone regions and $\mathrm{CH}$-stretching/HCH-bending combination regions between 1500 and $850 \mathrm{~nm}\left(6700-12000 \mathrm{~cm}^{-1}\right)$ (near-infrared (NIR) region), with the aim of adding new information on these molecules and possibly to tie it to some of the chiroptical properties explored in the literature. ${ }^{1-12}$ We had already published in the past some NIR-VCD data for $\mathbf{1}$ and $\mathbf{2}$ and for some related compounds. ${ }^{13,14}$ However, we have recently improved our apparatus for measuring NIR-VCD, obtaining better VCD data in the overtone region at $\Delta v=3$, new VCD data at $\Delta v=4$ for fenchone, and VCD data in the yet unexplored regions of $\mathrm{CH}$-stretchings/ $\mathrm{HCH}$-bendings combinations. We will complement our studies with the consideration of VCD and absorption data in the region 2800-7000 $\mathrm{cm}^{-1}$ $(3600-1500 \mathrm{~nm})$ from the literature ${ }^{15}$ or from some of our previous unpublished experiments, the results of which we are going to report here. We need to mention that quite recently Nafie's group has obtained nice NIR-VCD spectra on camphor and related compounds. ${ }^{16}$ An empirical analysis of all our data for both camphor and fenchone allows one to conjecture on which $\mathrm{CH}$-stretchings are the most important ones in generating $\mathrm{VCD}$ in the overtone regions. In the last part of the paper we report on two sorts of calculations. On the basis of the local mode hypothesis, ${ }^{17,18}$ we propose a heuristic protocol that allows one to calculate the VCD and absorption spectra as the superposition of the $16 \mathrm{VCD}$ and absorption spectra of the 16 deuterated species of camphor and fenchone, where just one $\mathrm{CH}$ is left undeuterated. The calculations for each isotopomer 
are obtained by the use of the GAUSSIAN package, ${ }^{19}$ exploiting the DFT approach. In our protocol, VCD and absorption intensities are calculated ab initio for $\Delta v=1$ transitions and are just transferred to the overtone region under study, whereas frequencies are built by Birge-Sponer relations, ${ }^{20}$ taking the harmonic part from the ab initio calculations and assuming a common anharmonicity value for all $16 \mathrm{CH}$-stretchings. Another model is also applied just for the calculation of dipole and rotational strengths, to verify the influence of the polarizability of the $\mathrm{C}=\mathrm{O}$ bond on the $\mathrm{CH}$-stretchings signals. We calculate the magnetic dipole moments as proposed for IR-VCD in some previous works ${ }^{21,22}$ that are adaptations of earlier works for UV circular dichroism (UV-CD) and optical rotation (OR). ${ }^{23-26}$ The two approaches are compared, by considering camphor and fenchone and also $(3 R)-(+)$-methylcyclopentanone and $(3 R)-$ $(+)$-methylcyclohexanone. VCD data of the latter compound in the infrared range has been analyzed several times; we mention here one of the most recent ab initio analyses by Devlin and Stephens. ${ }^{27}$

\section{Experimental/Material and Methods}

(a) Description of Instruments. The instrument that we employed for recording NIR-VCD spectra is a modified version of the one that we described in ref 14 . We used here an InGaAs detector cooled by the Peltier effect (down to $-10^{\circ} \mathrm{C}$ ), which we bought from Electro Optical System (EOS). Also, a new sample compartment was added between the monochromator and linear polarizer to take absorption baseline (ABL) spectra. The first modification allows one to get rid of thermal fluctuations and to obtain considerably flatter average spectra for an enatiomeric pair, with respect to our earlier experiments. ${ }^{14}$ The second change is essential for getting rid of artifacts connected to the absorption features, and its importance had been already pointed out in ref 28 .

We also recorded absorption spectra of fenchone in the IR region up to $7000 \mathrm{~cm}^{-1}$ on a JASCO FTIR 470-plus spectrometer $\left(4 \mathrm{~cm}^{-1}\right.$ resolution). The IR-VCD spectra presented in Figures $1 \mathrm{~A}$ and 8 were recorded at the University of Minnesota in the laboratory of the late Professor A. Moscowitz's group, and the apparatus has been described several times in the literature. $^{28}$

(b) Description of Measurements. The NIR-VCD spectra reported here were obtained as follows: we consider the difference between the CD spectra recorded with the sample placed between the photoelastic modulator and detector and the CD spectra in the so-called ABL position, namely, with the sample placed before the linear polarizer. Finally we divided the latter result by the DC signal obtained with the same apparatus. In most cases we took from 2 to 10 scans for each spectrum. When possible, we examined both enantiomers, to verify that mirror-image spectra be obtained. The samples of fenchones and camphors were purchased from Aldrich and were used as obtained without further purification.

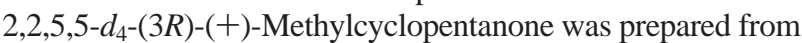
$(3 R)-(+)$-methylcyclopentanone, using the procedure outlined in refs 29 and 30. The samples were either neat liquids or $\mathrm{CCl}_{4}$ solutions. We employed quartz cells with $5-\mathrm{mm}$ path length for measurements between 1500 and $1100 \mathrm{~nm}$ and $10-$ or $5-\mathrm{cm}$ cells for measurements between 1100 and $800 \mathrm{~nm}$. (see figure captions for further details).

\section{Results and Discussion}

(a) Empirical Correlations. In Figure 1 we report the absorption and VCD spectra (when available) for both enantiomers of fenchone in the spectroscopic regions that are described below and are designated by the collective labels $v_{\mathrm{s}}$ for the $\mathrm{CH}$-stretching quantum number and $v_{\mathrm{b}}$ for the $\mathrm{HCH}$ bending quantum number: $2800-3100 \mathrm{~cm}^{-1}$ corresponding to $\left(\Delta v_{\mathrm{s}}=1, \Delta v_{\mathrm{b}}=0\right)$ (apart from possible Fermi resonance contributions with $\Delta v_{\mathrm{s}}=0, \Delta v_{\mathrm{b}}=2$ states); $5550-6150 \mathrm{~cm}^{-1}$ corresponding to $\left(\Delta v_{\mathrm{s}}=2, \Delta v_{\mathrm{b}}=0\right) ; 1300-1100 \mathrm{~nm}$ corresponding to $\left(\Delta v_{\mathrm{s}}=3, \Delta v_{\mathrm{b}}=0\right) ; 1000-850 \mathrm{~nm}$ corresponding to $\left(\Delta v_{\mathrm{s}}=4, \Delta v_{\mathrm{b}}=0\right)$; and $3950-4550 \mathrm{~cm}^{-1}$ corresponding to $\left(\Delta v_{\mathrm{s}}=1, \Delta v_{\mathrm{b}}=1\right) ; 1500-1300 \mathrm{~nm}$ corresponding to $\left(\Delta v_{\mathrm{s}}=2, \Delta v_{\mathrm{b}}=1\right) ; 1100-950 \mathrm{~nm}$ corresponding to $\left(\Delta v_{\mathrm{s}}=3, \Delta v_{\mathrm{b}}=1\right)$. For camphor the corresponding data for the regions $\left(\Delta v_{\mathrm{s}}=2, \Delta v_{\mathrm{b}}=1\right)$ and further are reported in Figure 2; the data for lower frequency regions, namely, for $\left(\Delta v_{\mathrm{s}}=1, \Delta v_{\mathrm{b}}=0\right)$, for $\left(\Delta v_{\mathrm{s}}=1, \Delta v_{\mathrm{b}}=1\right)$, and for $\left(\Delta v_{\mathrm{s}}=2\right.$, $\left.\Delta v_{\mathrm{b}}=0\right)$ can be found in ref 15 .

Let us first consider the absorption spectra of pure overtones $\left(\Delta v_{\mathrm{s}}=1-4 ; \Delta v_{\mathrm{b}}=0\right)$. The absorption spectra at $\Delta v_{\mathrm{s}}=3,4$ are remarkably similar for fenchone and camphor and consist of just one fairly narrow band with few shoulders, indicating that it is due to pure overtone states with all $v_{\mathrm{s}}$ quanta on isolated $\mathrm{CHs}$, which are vibrating independently of each other and have more or less the same frequency. Also the spectra at $\Delta v_{\mathrm{s}}=1$ are rather similar for the two molecules, consisting of a strong absorption band on the high-frequency side (ca. 2960 $\mathrm{cm}^{-1}$ ) in correspondence of $\mathrm{CH}_{3}$ and $\mathrm{CH}_{2}$ antisymmetric normal modes, ${ }^{15,30,31}$ a prominent shoulder at ca. $2930 \mathrm{~cm}^{-1}$, and a weaker band at ca. $2880 \mathrm{~cm}^{-1}$, in correspondence of $\mathrm{CH}_{3} / \mathrm{CH}_{2}$ symmetric normal modes. Fermi resonance contributions to these features are possible. The similarity in the absorption spectra of fenchone and camphor is not so striking at $\Delta v_{\mathrm{s}}=2$, but it is still quite notable (compare the data of Figure 1 and those in ref 15). For both molecules one observes two bands, the one at lower frequency corresponding to pure first overtone transitions to $(0, \ldots, 2, \ldots, 0)$-type states, the one at higher frequency corresponding to transition to $(0, \ldots, 1, \ldots, 1, \ldots, 0)$-type combination states. In passing we remind that in many cases the band at lower frequency in this region, which corresponds to a pure overtone in a local mode picture, has been observed not to be the most intense. ${ }^{32}$ In fact this region has intermediate characteristics between the local mode regime and the normal mode regime.

On this basis one can consider in Figure 3 the Birge-Sponer plots $^{20}$ for $\mathbf{1}$ and $\mathbf{2}$, where we report as dots the observed frequencies $\omega_{v_{\mathrm{s}}}$ divided by the stretching overtone order $v_{\mathrm{s}}$ versus $v_{\mathrm{s}}$. Since in a simplified local oscillator model, $\mathrm{CHs}$ are thought to vibrate independently of one another, one may assume ${ }^{17,18}$

$$
\omega_{v_{\mathrm{s}}}=\left(\omega_{\mathrm{s}}^{0}-\chi_{\mathrm{s}}\right) v_{\mathrm{s}}-\chi_{\mathrm{s}} v_{\mathrm{s}}^{2}
$$

Thus one may derive the values of the harmonic frequency $\omega_{\mathrm{s}}{ }^{0}$ and of the anharmonicity $\chi_{\mathrm{s}}$ by least-squares fitting of the lowest frequency value observed in each overtone region (pure local modes). We report such values in Table 1 and compare them with the values previously derived on the basis of similar studies for cyclohexanone, ${ }^{33}$ cyclopentanone, ${ }^{33}$ and cyclopentane..$^{34,35}$ The three latter molecules are the three ring structures that compose $\mathbf{1}$ and $\mathbf{2}$, despite differences in the ring conformations. It is remarkable that, in the limits of the accuracy of the present study and with only one exception, there is a strong similarity in the mechanical frequencies of the $\mathrm{CHs}$ of cyclohexanone, cyclopentanone, and cyclopentane with the $\mathrm{CHs}$ of fenchone and camphor. We remind, e.g., that cyclohexane has quite different values for $\omega_{\mathrm{s}}{ }^{0}\left(\omega_{\mathrm{s}}{ }^{0}=2975\right.$ and $2999 \mathrm{~cm}^{-1}$ for axial and equatorial $\mathrm{CHs}$, respectively ${ }^{34}$ ). This leads us to 

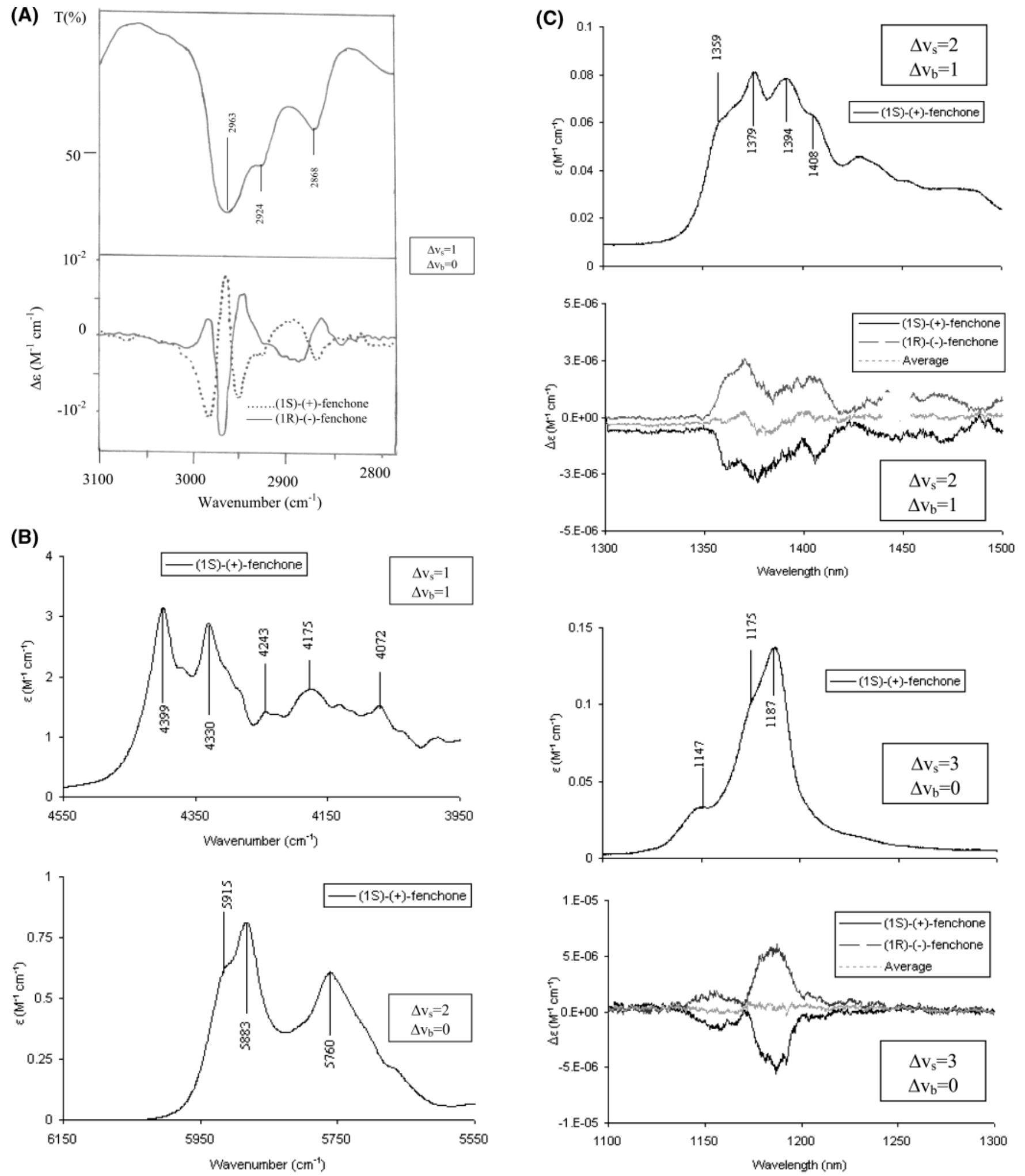

Figure 1. Absorption and vibrational circular dichroism spectra in the infrared and near-infrared for the two enantiomers of fenchone. (A) Absorption and VCD spectra at $\Delta v_{\mathrm{s}}=1, \Delta v_{\mathrm{b}}=0$ (recorded at the University of Minnesota); 0.060-cm path length $\mathrm{CaF}_{2} \mathrm{cell}_{\text {for }} 0.05 \mathrm{M} \mathrm{CCl}_{4}$ solutions. (B) Absorption spectrum at $\Delta v_{\mathrm{s}}=1, \Delta v_{\mathrm{b}}=1$ (top); absorption spectrum at $\Delta v_{\mathrm{s}}=2, \Delta v_{\mathrm{b}}=0$ (bottom) both taken with Jasco FTIR 470 plus instrument for neat liquid in a 0.2-mm quartz cell. (C) Absorption and VCD spectra at $\Delta v_{\mathrm{s}}=2, \Delta v_{\mathrm{b}}=1$ (top) and absorption and VCD spectra at $\Delta v_{\mathrm{s}}=3$, $\Delta v_{\mathrm{b}}=0$ (bottom) both for neat liquid in a $0.5-\mathrm{cm}$ quartz cell. (D) Absorption spectra at $\Delta v_{\mathrm{s}}=3, \Delta v_{\mathrm{b}}=1$ (top) for neat liquid in a 5-cm quartz cell and absorption and VCD spectra at $\Delta v_{\mathrm{s}}=4, \Delta v_{\mathrm{b}}=0$ (bottom) for neat liquid in a 10-cm quartz cell. In parts $\mathrm{C}$ and $\mathrm{D}$ the reported average VCD spectra provide a sort of confidence criteria. 


\section{(D)}
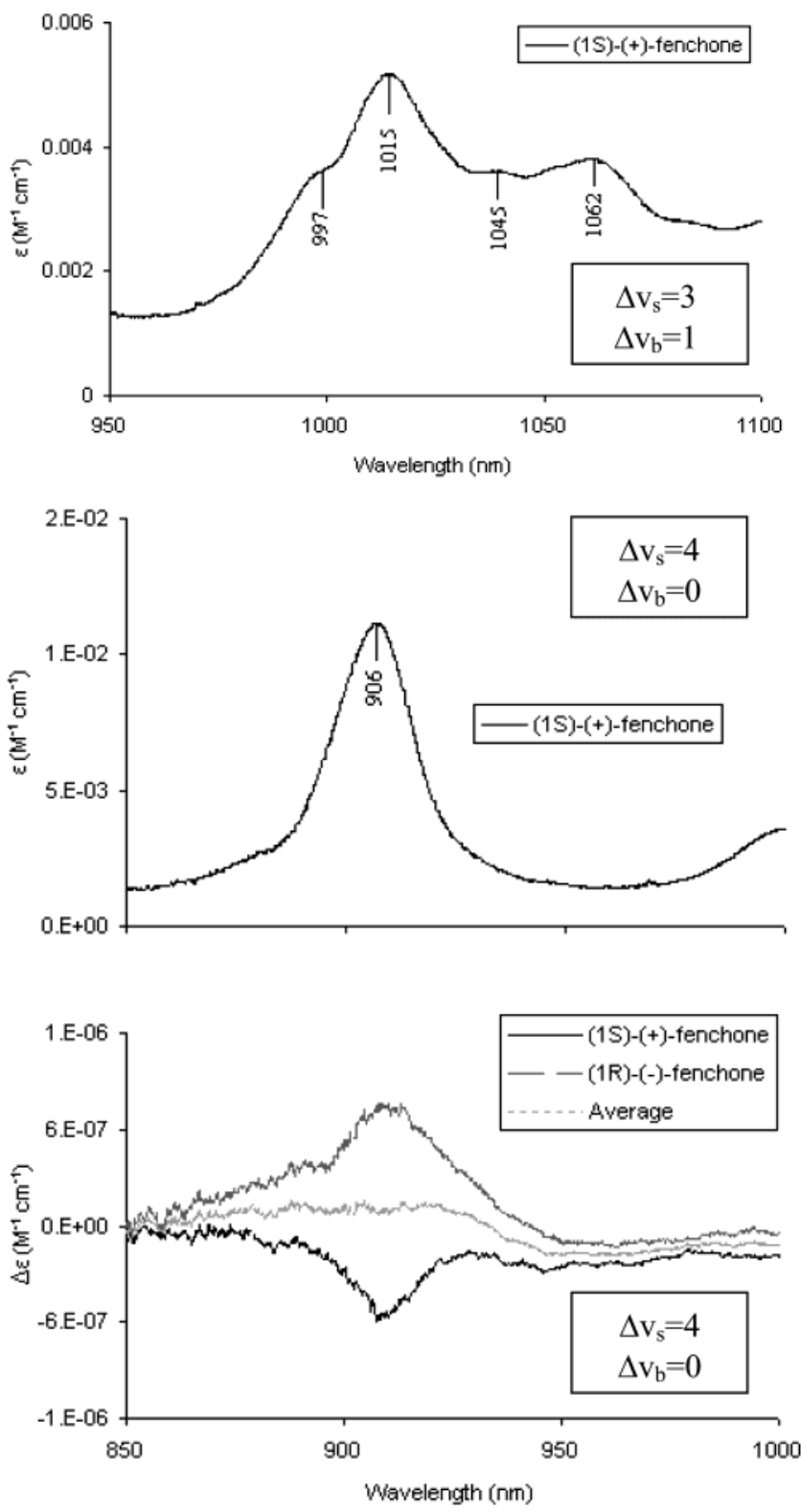

Figure 1. Continued.

conclude that the observed fact that the $\Delta v_{\mathrm{s}}=3,4$ regions are composed of just one narrow absorption band has its explanation in very similar $\mathrm{CH}$ bond stretchings in all the three molecular moieties. We also observe that the Birge-Sponer line drawn in Figure 3 according with the values of $\omega_{\mathrm{s}}{ }^{0}$ and $\chi_{\mathrm{s}}$ reported in Table 1 passes very close to the value of $\omega_{v_{\mathrm{s}}} / v_{\mathrm{s}}$ for the central shoulder in the region $\Delta v_{\mathrm{s}}=1$.

Now let us consider the absorption spectra of combination modes regions $\left(\Delta v_{\mathrm{s}}=1-3, \Delta v_{\mathrm{b}}=1\right)$. Also here we notice a strongly similar behavior of the two molecules in the three regions. The interpretation of the combination spectra though is much more difficult than for the pure overtones spectra. We notice that in all the three regions there is a broad absorption continuum at low wavenumbers/high wavelengths. At high wavenumbers/low wavelengths there is a more or less resolved doublet with shoulders for $\Delta v_{\mathrm{s}}=1$ and 2 , which becomes a single prominent peak with a shoulder on the high-frequency side at $\Delta v_{\mathrm{s}}=3$. Following a Dunham-type expansion, ${ }^{20}$ for the energies $E_{v_{\mathrm{s}}, v_{\mathrm{b}}}$ for $\mathrm{CH}$-stretching/HCH-bending combination modes, one obtains

$$
\begin{aligned}
E_{v_{\mathrm{s}}, v_{\mathrm{b}}}=\omega_{\mathrm{s}}{ }^{0}\left(v_{\mathrm{s}}+\frac{1}{2}\right)- & \chi_{\mathrm{s}}\left(v_{\mathrm{s}}+\frac{1}{2}\right)^{2}+\omega_{\mathrm{b}}{ }^{0}\left(v_{\mathrm{s}}+\frac{1}{2}\right)- \\
& \chi_{\mathrm{b}}\left(v_{\mathrm{b}}+\frac{1}{2}\right)^{2}-\chi_{\mathrm{sb}}\left(v_{\mathrm{s}}+\frac{1}{2}\right)\left(v_{\mathrm{b}}+\frac{1}{2}\right)
\end{aligned}
$$

An acceptable simplifying hypothesis is that the more intense and well-resolved peaks in the combination regions (Figures $1 \mathrm{~B}-\mathrm{D}$ and $2 \mathrm{~A}-\mathrm{B})$ are due to states with $v_{\mathrm{b}}=1$. For small values of $\chi_{\mathrm{sb}}$ the differences between the observed frequencies in contiguous regions of combinations and pure overtones, namely $\omega_{v_{\mathrm{s}}, v_{\mathrm{b}}=1}-\omega_{v_{\mathrm{s}}, v_{\mathrm{b}}=0}$, is close to a bending fundamental, among the 14 ones, observed for fenchone and camphor. ${ }^{5}$ Indeed nearly the same bending frequency value (ca. $1435 \mathrm{~cm}^{-1}$ ) is obtained (a) if one considers the most intense frequency peak at $v_{\mathrm{s}}=3, v_{\mathrm{b}}=1$ minus the overtone frequency $v_{\mathrm{s}}=3, v_{\mathrm{b}}=0$; (b) the lower frequency of the two most intense peaks in the region $v_{\mathrm{s}}=2, v_{\mathrm{b}}=1$ minus the overtone frequency $v_{\mathrm{s}}=2, v_{\mathrm{b}}$ $=0$, which was attributed to $(0, \ldots, 2, \ldots, 0)$ local modes; (c) the higher frequency observed in the region $v_{\mathrm{s}}=1, v_{\mathrm{b}}=1$ minus the antisymmetric mode frequency in the $v_{\mathrm{s}}=1, v_{\mathrm{b}}=0$ region (indeed antisymmetric stretching modes and bending modes can be considered independent of each other for symmetry reasons). Moreover, taking the difference $E_{v_{\mathrm{s}}, 1}-E_{0,1}$, one obtains

$$
\omega_{v_{\mathrm{s}}, v_{\mathrm{b}}=1}-\omega_{v_{\mathrm{s}}=0},_{\mathrm{b}}=1=\left(\omega_{\mathrm{s}}{ }^{0}-\chi_{\mathrm{s}}-\frac{3}{2} \chi_{\mathrm{sb}}\right) v_{\mathrm{s}}-\chi_{\mathrm{s}} v_{\mathrm{s}}^{2}
$$

Equation 3 is similar to eq 1 . Inserting $\omega_{v_{\mathrm{s}}=0, v_{\mathrm{b}}=1}=1435$ $\mathrm{cm}^{-1}$ as representative of the $\mathrm{HCH}$-bending frequencies, a plot of $\left(\omega_{v_{\mathrm{s}}, v_{\mathrm{b}}=1}-\omega_{v_{\mathrm{s}}=0, v_{\mathrm{b}}=1}\right) / v_{\mathrm{s}}$ versus $v_{\mathrm{s}}$ gives indeed a straight line parallel to that of $\omega_{\mathrm{s}} / v_{\mathrm{s}}$ versus $v_{\mathrm{s}}$ (same slope $\chi_{\mathrm{s}}$ and slightly different intercept with the ordinate axis by $-3 / 2 \chi_{\mathrm{sb}}$ ) for the most intense peaks in the three combination regions (at lowest wavelength/highest wavenumber). This analysis shows that the most intense peaks in the combination regions are assignable, in a rough approximation, to $v_{\mathrm{s}}$ quanta of pure $\mathrm{CH}$-stretching local modes plus one quantum of $\mathrm{HCH}$-bending; the perturbation brought in by all other possible combinations may explain the broad continuum and possibly the splittings, but will be ignored here. This gives us a first clue to the interpretation of the VCD spectra of this previously unexplored region.

Let us now turn to the analysis of the VCD spectra of pure $\mathbf{C H}$-stretching overtones. If one considers the VCD spectra for $\Delta v_{\mathrm{s}}=3,4$ in Figures 1 and 2, one notices the following facts: (i) The VCD spectra for camphor are intrinsically weaker than the corresponding ones of fenchone by approximately a factor of 2. (ii) At $\Delta v_{\mathrm{s}}=3$ the VCD spectrum of fenchone (Figure 1C) consists of two broad bands with the same sign, the most intense one sitting exactly under the major absorption. Instead, camphor (Figure 2A) shows a clear bisignate couplet, in correspondence of the major absorption peak, the lower wavelength band of the couplet being more intense. In addition, a third VCD band of alternating sign appears at still lower wavelength (see also ref 16). (iii) At $\Delta v_{\mathrm{s}}=4$ the structuring evidenced in the VCD spectrum of fenchone at $\Delta v_{\mathrm{s}}=3$ is lost, possibly as a result of low efficiency of our apparatus there, and only one VCD band remains in correspondence of the major absorption band, correlating in sign with the datum at $\Delta v_{\mathrm{s}}=3$ (Figure 1D). For camphor, the triplet of signed VCD bands of the preceding region is observed also here in the same order, but we point out that the signal-to-noise ratio in this region is not very good (Figure 2B). (iv) Finally the most intense VCD band has the same sign for (-)-camphor as for (-)-fenchone, 
(A)
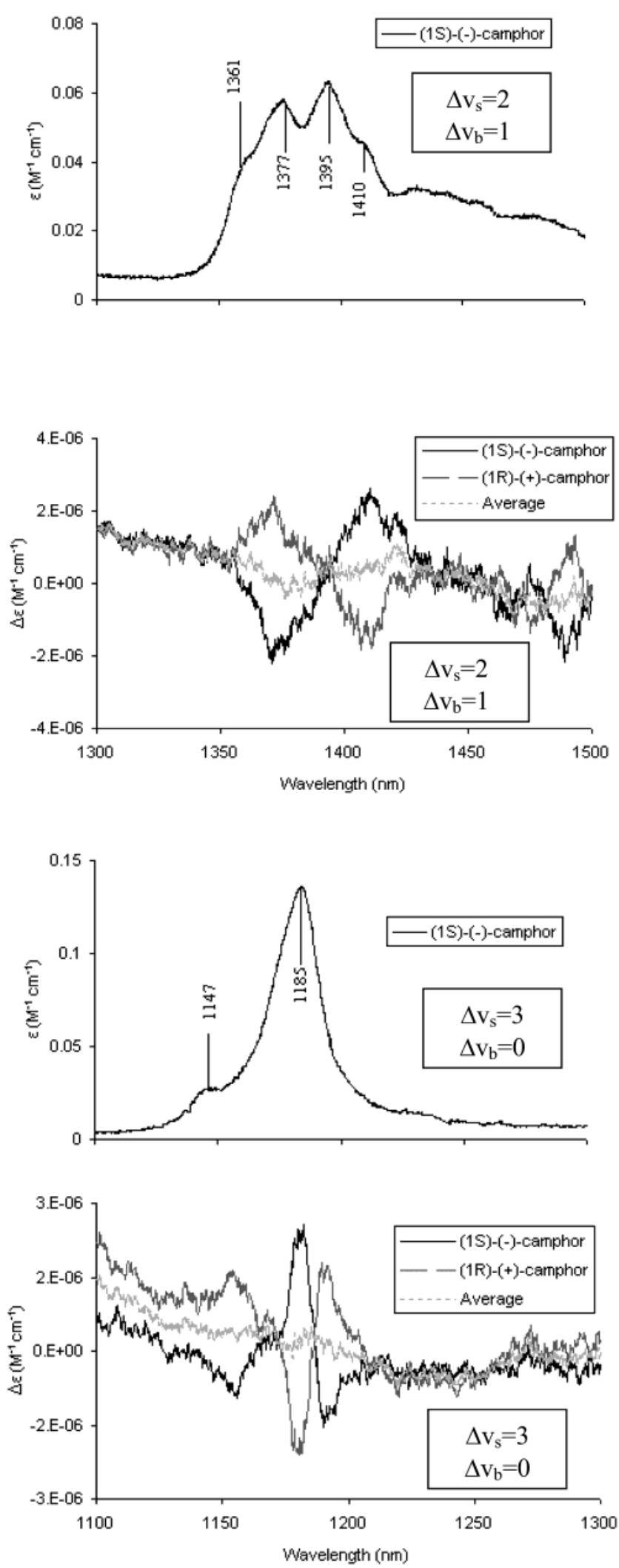

(B)
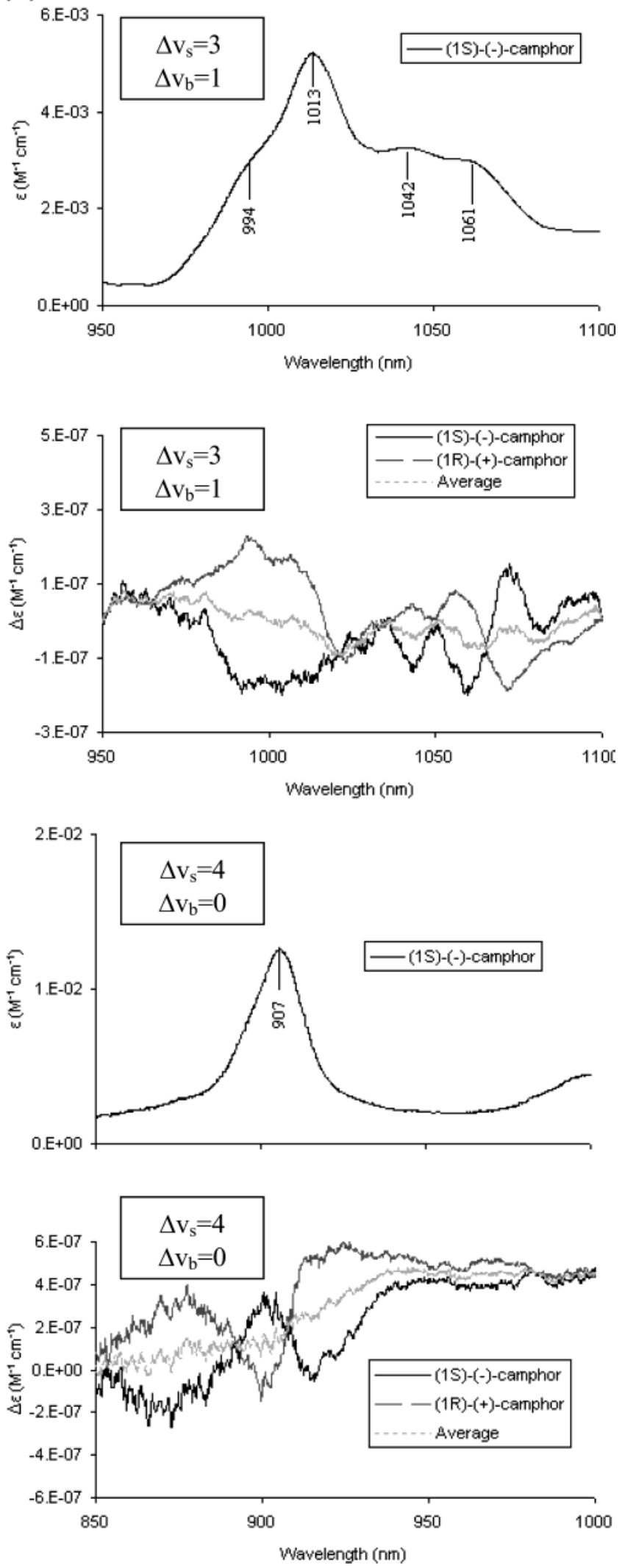

Figure 2. Absorption and vibrational circular dichroism spectra in the near-infrared for the two enantiomers of camphor. (A) Absorption and VCD spectra at $\Delta v_{\mathrm{s}}=2, \Delta v_{\mathrm{b}}=1$ (top) and absorption and VCD spectra at $\Delta v_{\mathrm{s}}=3, \Delta v_{\mathrm{b}}=0$ (bottom), both for $3 \mathrm{M}$ solutions in CCl in a 0.5 -cm quartz cell. (B) Absorption and VCD spectra at $\Delta v_{\mathrm{s}}=3, \Delta v_{\mathrm{b}}=1$ for $3 \mathrm{M}$ solution in $\mathrm{CCl}_{4}$ in a 5-cm quartz cell (top) and absorption and VCD spectra at $\Delta v_{\mathrm{s}}=4, \Delta v_{\mathrm{b}}=0$ for $3 \mathrm{M}$ solution in $\mathrm{CCl}_{4}$ in a 10 -cm quartz cell (bottom). In both cases also average VCD spectra are provided.

which have opposite absolute configuration (AC) at stereogenic carbons 1 and 4 (see the structures above). At $\Delta v_{\mathrm{s}}=2$ we have just the VCD data for camphor from ref 15 . We notice that the major VCD feature is in correspondence with the absorption band that has been assigned above to the pure first overtone and correlates in sign with the VCD data at $\Delta v_{\mathrm{s}}=3$. Finally at 

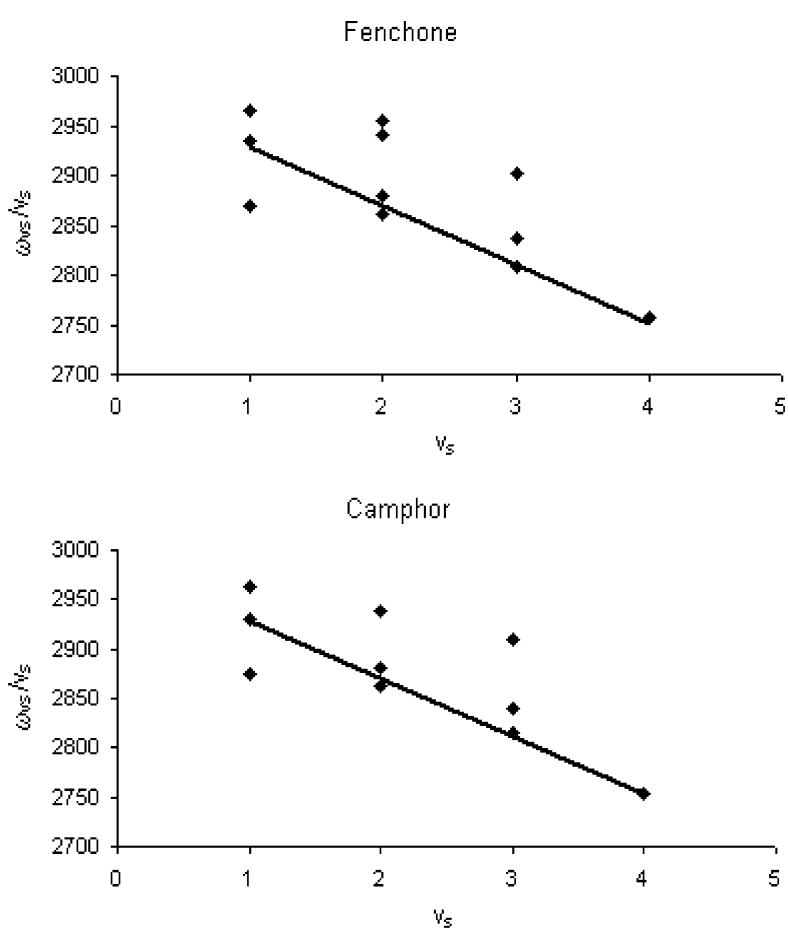

Figure 3. Birge-Sponer plots for the absorption features of fenchone (top) and camphor (bottom). For fenchone we used the experimental frequencies of the present work. For camphor we utilized data of the present work plus data from ref 15.

$\Delta v_{\mathrm{s}}=1$ one has five VCD bands, which alternate in sign for fenchone (this work) and for camphor, ${ }^{15}$ except for the two minor features at lowest frequency in camphor. Except for the latter features, the observed VCD bands for $(1 S)-(-)$-camphor and $(1 R)-(-)$-fenchone have the same sign, and similar peak position and intensity. It is interesting to notice that the VCD band in the fundamental region at about $2930 \mathrm{~cm}^{-1}$, in correspondence to the absorption shoulder that was seen above to correlate with the major absorption peaks at higher overtone order via the Birge-Sponer line, has the same sign as the major VCD band at $\Delta v_{\mathrm{s}}=2-4$. As noticed above, all the $\mathrm{CH}-$ stretching modes produce absorption signals in fairly narrow regions of each overtone order, so it is hard to identify the $\mathrm{CH}$ stretchings that are responsible for the vibrational optical activity or at least to reduce the number of $\mathrm{CHs}$ on the basis of simple band separation. Though if one takes into account that the VCD spectra of the enantiomers of fenchone and camphor with the same AC are opposite for almost each single band at $\Delta v_{\mathrm{s}}=1$ and for the sum of all VCD bands at $\Delta v_{\mathrm{s}}=3$ (and possibly $\left.\Delta v_{\mathrm{s}}=4\right)$, one is tempted to conclude that the vibrational optical activity is mostly generated in a molecular moiety of opposite configuration in the two enantiomers of camphor and fenchone with the same AC. Among the $\mathrm{CH}$ bonds of the three rings,<smiles>CC12CC[C@H](C1)C(C)(C)C2=O</smiles><smiles>CC12CCC(CC1=O)C2(C)C</smiles>

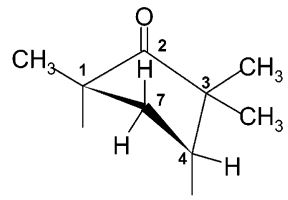

(1S)-(+)-fenchone

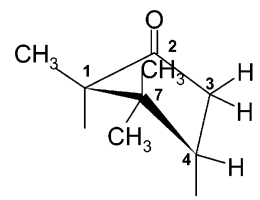

(1S)-(-)-camphor
Figure 4. Chemical structures of $(1 S)-(+)$-fenchone and $(1 S)-(-)-$ camphor and geometries of the cyclopentanone moieties thereof (see text).

just one fragment exists with opposite chirality relative to the $\mathrm{C}=\mathrm{O}$ bond in the two molecules; it is located within the cyclopentanone moiety and contains three $\mathrm{CH}$ bonds, namely, the $\mathrm{CH}_{2} \mathrm{CH}$ fragment for carbons 7 and 4 of (+)-fenchone AND the $\mathrm{CH}_{2} \mathrm{CH}$ fragment for carbons 3 and 4 of $(-)$-camphor (see Figure 4). Also the fragments defined by the three methyls in the two molecules have opposite configurations in the two molecules. All of the other fragments in the other two moieties of the two molecules, namely, in the cyclohexanone and cyclopentane moieties, have the same chirality for the two molecules. The fact that the cyclopentanone moiety is important for optical activity had been previously pointed out in ref 4 , in interpreting the $\mathrm{CD}$ signals of the two lowest electronic transitions, namely, the $n \rightarrow \pi^{*}$ and the one with next higher frequency; therein it had already been noticed as an important fact that both electronic transitions are of opposite sign for the same AC of camphor and fenchone. In any case our empirical correlations need to be confirmed by ab initio or at least by model calculations; an attempt in that direction will be presented below.

Let us finally shortly comment on the VCD spectra of bending-stretching combination regions. For fenchone, we notice from Figure 1C that the VCD band in correspondence with the major absorption peak has the same sign for the region $\left(\Delta v_{\mathrm{s}}=2, \Delta v_{\mathrm{b}}=1\right)$ that we have investigated with respect to the $\left(\Delta v_{\mathrm{s}}=3, \Delta v_{\mathrm{b}}=0\right)$ region. For the region $\left(\Delta v_{\mathrm{s}}=3, \Delta v_{\mathrm{b}}=\right.$ 1) we do not have good enough data. For camphor, the data for the $\left(\Delta v_{\mathrm{s}}=1, \Delta v_{\mathrm{b}}=1\right)$ region from ref 15 shows the VCD feature in correspondence with the major absorption bands. For the further regions $\left(\Delta v_{\mathrm{s}}=2, \Delta v_{\mathrm{b}}=1\right)$ and $\left(\Delta v_{\mathrm{s}}=3, \Delta v_{\mathrm{b}}=1\right)$ (Figure 2A and $\mathrm{B}$ ) this is still observed. In contrast to the absorption data, the VCD data are hard to rationalize for these regions; we have reported them just for completeness.

(b) Calculations. One of the findings of the present study is the sign difference for the overall VCD for each overtone or

TABLE 1: Comparison of Harmonic Frequencies $\omega_{\mathrm{s}}{ }^{0}$ and Anharmonicities $\chi_{\mathrm{s}}$ for Camphor and Fenchone, Experimentally Determined from Plots of Figure 3, with the Corresponding Values Determined in References 33-35 for Cylopentanone, Cyclohexanone, and Cyclopentane ${ }^{a}$

\begin{tabular}{|c|c|c|c|c|c|c|c|c|c|c|}
\hline & \multirow{2}{*}{$\begin{array}{l}\text { camphor } \\
\text { (this work) }\end{array}$} & \multirow{2}{*}{$\begin{array}{l}\text { fenchone } \\
\text { (this work) }\end{array}$} & & \multicolumn{2}{|c|}{$\begin{array}{l}\text { cyclopentanone } \\
\text { (ref 33) }\end{array}$} & & \multicolumn{2}{|c|}{$\begin{array}{l}\text { cyclohexanone } \\
\text { (ref 33) }\end{array}$} & \multicolumn{2}{|c|}{$\begin{array}{l}\text { cyclopentane } \\
\text { (refs 34,35) }\end{array}$} \\
\hline & & & & $\mathrm{ax}$ & eq & & $\mathrm{ax}$ & eq & $\mathrm{ax}$ & eq \\
\hline$\omega_{\mathrm{s}}^{0}\left(\mathrm{~cm}^{-1}\right)$ & 3062 & 3063 & $\alpha$ & 3065 & 3085 & $\alpha$ & 3000 & 3070 & 3047 & 3049 \\
\hline$\chi_{\mathrm{s}}\left(\mathrm{cm}^{-1}\right)$ & 62 & 62 & & 67 & 61 & & 62 & 63 & 67 & 67 \\
\hline$\omega_{\mathrm{s}}^{0}\left(\mathrm{~cm}^{-1}\right)$ & & & $\beta, \gamma$ & 3065 & 3097 & $\beta, \gamma$ & 3062 & 3062 & 3075 & 3061 \\
\hline$\chi_{\mathrm{s}}\left(\mathrm{cm}^{-1}\right)$ & & & & 67 & 65 & & 67 & 67 & 65.3 & 63.1 \\
\hline
\end{tabular}

${ }^{a}$ For the latter cases different experimental features were assigned to axial and equatorial $\mathrm{CH}$ bonds in the molecular ring, and eventually further distinction by the distance from the $\mathrm{C}=\mathrm{O}$ bond ( $\alpha$ or $\beta$ position) is given. 
TABLE 2: (3R)-(+)-Methylcyclopentanone (Half-Chair Conformer with $\mathrm{CH}_{3}$ in Both Equatorial and Axial Positions) ${ }^{a}$

\begin{tabular}{|c|c|c|c|c|c|c|c|c|}
\hline \multirow[b]{2}{*}{$\begin{array}{l}\text { groups and } \\
\text { assignment }^{b}\end{array}$} & \multicolumn{4}{|c|}{$(3 R)-(+)$-methylcyclopentanone; equatorial $\mathrm{CH}_{3}$} & \multicolumn{4}{|c|}{$(3 R)-(+)$-methylcyclopentanone; axial $\mathrm{CH}_{3}$} \\
\hline & $\omega_{\mathrm{s}}^{0}\left(\mathrm{~cm}^{-1}\right)$ & $\lambda\left(\Delta v_{\mathrm{s}}=3\right)(\mathrm{nm})$ & $R$ ab initio & $\begin{array}{c}R\left(\Delta v_{\mathrm{s}}=3\right) \\
\text { pol. model }\end{array}$ & $\omega_{\mathrm{s}}{ }^{0}\left(\mathrm{~cm}^{-1}\right)$ & $\lambda\left(\Delta v_{\mathrm{s}}=3\right)(\mathrm{nm})$ & $R$ ab initio & $\begin{array}{c}R\left(\Delta v_{\mathrm{s}}=3\right) \\
\text { pol. model }\end{array}$ \\
\hline $\mathrm{CH}_{2}-\alpha$ peq & 3109.6 & 1178.0 & 6.46 & 2.22 & 3107.2 & 1179.0 & -6.36 & -2.28 \\
\hline peq & 3109.2 & 1178.2 & 4.78 & 2.09 & 3105.2 & 1179.8 & -6.39 & -2.09 \\
\hline pax & 3050.0 & 1203.0 & -3.22 & -2.6 & 3059.3 & 1199.3 & 1.36 & 2.6 \\
\hline pax & 3029.5 & 1212.3 & -1.29 & -2.57 & 3046.8 & 1204.8 & 2.38 & 2.59 \\
\hline $\mathrm{C}^{*} \mathrm{H}$ ax & 3017.5 & 1217.6 & -5.48 & 0.44 & 3064.4 & 1197.1 & -6.18 & 0.47 \\
\hline $\mathrm{CH}_{2}-\beta$ eq & 3094.1 & 1184.5 & 5.55 & -0.4 & 3092.1 & 1185.4 & -5.89 & 0.4 \\
\hline ax & 3047.5 & 1204.5 & -5.51 & 0.47 & 3063.9 & 1197.4 & 3.23 & -0.47 \\
\hline $\mathrm{CH}_{3}$ & 3091.0 & 1185.8 & 6.21 & 0.21 & 3091.1 & 1185.8 & 2.57 & 0.6 \\
\hline & 3086.2 & 1187.8 & -3.86 & 0.08 & 3089.8 & 1186.3 & -3.81 & -0.34 \\
\hline & 3075.4 & 1192.4 & -0.31 & -0.2 & 3076.9 & 1191.8 & 1.99 & 0.02 \\
\hline
\end{tabular}

${ }^{a}$ Assignment (1st column), ab initio calculated frequencies for the fundamental ( $\Delta v=1,2$ nd and 6 th columns), calculated wavelengths for the 2nd overtone $\left(\Delta v=3,3 \mathrm{rd}\right.$ and 7 th columns) as proposed in the text. Calculated rotational strengths out of GAUSSIAN for $\Delta v=1$ in $10^{-44}$ esu ${ }^{2}$ $\mathrm{cm}^{2}$ units (4th and 8th columns) and based on the classical model presented in the text (5th and 9th columns) in $10^{-47} \mathrm{esu}^{2} \mathrm{~cm}^{2}$ units. ${ }^{b}$ pax and peq mean pseudoaxial and pseudoequatorial, respectively; ax and eq mean truly axial and truly equatorial, respectively.

TABLE 3: (3R)-(+)-Methylcyclohexanone (Chair Conformer with $\mathrm{CH}_{3}$ in Both Equatorial and Axial Positions) ${ }^{a}$

\begin{tabular}{|c|c|c|c|c|c|c|c|c|}
\hline \multirow{2}{*}{$\begin{array}{l}\text { groups and } \\
\text { assignment }\end{array}$} & \multicolumn{4}{|c|}{$(3 R)-(+)$-methylcyclohexanone; equatorial $\mathrm{CH}_{3}$} & \multicolumn{4}{|c|}{$(3 R)-(+)$-methylcyclohexanone; axial $\mathrm{CH}_{3}$} \\
\hline & $\overline{\omega_{\mathrm{s}}^{0}\left(\mathrm{~cm}^{-1}\right)}$ & $\lambda\left(\Delta v_{\mathrm{s}}=3\right)(\mathrm{nm})$ & $R$ ab initio & $R$ pol. model & $\overline{\omega_{\mathrm{s}}^{0}\left(\mathrm{~cm}^{-1}\right)}$ & $\lambda\left(\Delta v_{\mathrm{s}}=3\right)(\mathrm{nm})$ & $R$ ab initio & $R$ pol. model \\
\hline $\mathrm{CH}_{2}-\alpha \mathrm{eq}$ & 3111.8 & 1177.1 & -2.92 & -0.49 & 3110.5 & 1177.7 & 3.38 & 0.43 \\
\hline eq & 3104.3 & 1180.2 & 2.8 & 0.44 & 3103.9 & 1180.4 & -3.56 & -0.35 \\
\hline $\mathrm{ax}$ & 3029.4 & 1212.4 & -9.26 & 2.22 & 3033.2 & 1210.7 & -11.73 & 2.18 \\
\hline $\mathrm{ax}$ & 3018.5 & 1217.2 & 9.26 & -2.22 & 3028.9 & 1212.6 & 10.01 & -2.2 \\
\hline $\mathrm{C}^{*} \mathrm{H}$ ax & 3011.5 & 1220.3 & -8.54 & 0.64 & 3035 & 1209.9 & -3.96 & 0.83 \\
\hline $\mathrm{CH}_{2}-\beta$ eq & 3071.9 & 1193.9 & -3.54 & 0.8 & 3070.9 & 1194.4 & 4.75 & -0.81 \\
\hline ax & 3048.8 & 1203.9 & 5.94 & -0.68 & 3065.3 & 1196.8 & 3.16 & 0.68 \\
\hline $\mathrm{CH}_{2}-\gamma \mathrm{eq}$ & 3063.1 & 1197.7 & -0.12 & 0.01 & 3063.2 & 1197.7 & 0.12 & -0.006 \\
\hline ax & 3024 & 1214.8 & 0.09 & -0.01 & 3036.2 & 1209.4 & -0.21 & 0.005 \\
\hline $\mathrm{CH}_{3}$ & 3091.6 & 1185.6 & 1.72 & -0.14 & 3094.1 & 1184.5 & 2.92 & 0.92 \\
\hline & 3089.3 & 1186.6 & -1.59 & 0.43 & 3089.2 & 1186.6 & 4.73 & -0.18 \\
\hline & 3073.7 & 1193.1 & 2.07 & -0.17 & 3087.6 & 1187.3 & -2.31 & -0.34 \\
\hline
\end{tabular}

${ }^{a}$ Assignment (1st column), ab initio calculated frequencies for the fundamental ( $\Delta v=1,2$ nd and 6th columns), calculated wavelengths for the 2nd overtone $\left(\Delta v=3,3 \mathrm{rd}\right.$ and 7 th columns) as proposed in the text. Calculated rotational strengths out of GAUSSIAN for $\Delta v=1$ in $10^{-44}$ esu ${ }^{2}$ $\mathrm{cm}^{2}$ units (4th and 8th columns) and based on the classical model presented in the text (5th and 9th columns) in $10^{-47} \mathrm{esu}^{2} \mathrm{~cm}^{2}$ units.

combination region for the two quite similar molecules $(1 S)$ $(+)$-fenchone and $(1 S)-(-)$-camphor, with the same absolute configuration. This can be clearly seen looking at the region $\left(\Delta v_{\mathrm{s}}=3, \Delta v_{\mathrm{b}}=0\right)$, where one can also appreciate that two broad unresolved VCD bands have the same sign for fenchone and three different VCD bands alternate in sign for camphor. Another striking sign inversion had been observed for $(3 R)$ (+)-methylcyclohexanone and $(3 R)-(+)$-methylcyclopentanone: 13,14 for the latter compound the VCD spectra were consistently observed for $\Delta v_{\mathrm{s}}=2,3,4, \Delta v_{\mathrm{b}}=0$ to exhibit a minus sign in the major VCD feature and for the former one a plus sign for the corresponding major VCD feature. This has stimulated us to check whether a common mechanism for the generation of vibrational optical activity in the overtone region exists in $\mathrm{C}=\mathrm{O}$ containing molecules, as hinted already in ref 14 . We have verified this conjecture by performing two types of calculations. The first type is based on ab initio calculations of frequencies, dipole strengths, and rotational strengths at $\Delta v_{\mathrm{s}}=$ 1 for the $16 d_{15}$-isotopomers of camphor and fenchone, namely, those isotopomers with one $\mathrm{CH}$ and $15 \mathrm{CD}$ bonds in all possible permutations, and the second type is based on ab initio calculations of frequencies and on model classical calculations of dipole and rotational strengths. (For $(3 R)-(+)$-methylcyclohexanone the considered isotopomers are the $d_{11}$ ones, and for $(3 R)-(+)$-methylcyclopentanone are the $d_{9}$ ones). Let us describe them shortly and report the corresponding results.

(b1) Approach based Only on ab Initio Calculations. The first approach is heuristic. It is well-known that local mode overtone spectroscopy gives information about mechanical frequencies similar to selective deuteration; ${ }^{17,36}$ on this basis we decided to use state-of-the-art calculations for fundamental transitions in deuterated molecules leaving only one undeuterated $\mathrm{CH}$ group at a time. We have used DFT calculations based on B3LYP functional ${ }^{37}$ and $6-31 G^{* *}$ basis set, ${ }^{38}$ as obtained by GAUSSIAN98. ${ }^{19}$ The fully optimized geometries have been taken into account. We have considered one conformation for the methyls. As for the conformation of the six- and fivemembered rings in $(3 R)-(+)$-methylcyclohexanone and $(3 R)$ $(+)$-methylcyclopentanone, respectively, we have considered the two chair or half-chair conformers with the methyl in the equatorial and axial position. The calculated energy separation between the lowest energy conformer (the one with the equatorial methyl) and the other one is $1.57 \mathrm{kcal} / \mathrm{mol}$ for $(3 R)$ $(+)$-methylcyclohexanone and $1.23 \mathrm{kcal} / \mathrm{mol}$ for $(3 R)-(+)-$ methylcyclopentanone, as per GAUSSIAN.

For calculating frequencies, dipole strengths, and rotational strengths we propose the following protocol:

(I) Frequencies. We take the values for $\omega_{\mathrm{s}}{ }^{0}$ for isolated $\mathrm{CH}$ frequencies obtained $\mathrm{ab}$ initio, without scaling factors, for the $16 d_{15}$-isotopomers of fenchone and camphor and for the 10 and $12 d_{9}$ - and $d_{11}$-isotopomers of $(3 R)-(+)$-methylcyclopentanone and $(3 R)-(+)$-methylcyclohexanone, respectively. All of these values are reported in column 2 of Tables $2-4$. We observe two important facts: first, the calculated frequencies for fenchone and camphor fall in a very narrow interval, and second, the calculated values for $\omega_{\mathrm{s}}{ }^{0}$ are not too different from the experimentally determined mechanical frequencies, being systematically higher by ca. $30 \mathrm{~cm}^{-1}$ (see Table 1). This result is 
TABLE 4: (1S)-(-)-Camphor and $(1 S)-(+)-$ Fenchone $^{a}$

\begin{tabular}{|c|c|c|c|c|c|c|c|c|c|}
\hline \multicolumn{5}{|c|}{$(1 S)$-(-)-camphor } & \multicolumn{5}{|c|}{$(1 S)-(+)$-fenchone } \\
\hline groups & $\omega_{\mathrm{s}}^{0}\left(\mathrm{~cm}^{-1}\right)$ & $\lambda\left(\Delta v_{\mathrm{s}}=3(\mathrm{~nm})\right.$ & $R$ ab initio & $R$ pol. model & groups & $\omega_{\mathrm{s}}^{0}\left(\mathrm{~cm}^{-1}\right)$ & $\lambda\left(\Delta v_{\mathrm{s}}=3(\mathrm{~nm})\right.$ & $R$ ab initio & $R$ pol. model \\
\hline $\mathrm{CH}^{*}$ in 4 & 3091.9 & 1185.4 & -1.05 & 0.20 & $\mathrm{CH}^{*}$ in 4 & 3090.8 & 1185.9 & -1.12 & 0.22 \\
\hline \multirow[t]{2}{*}{$\mathrm{CH}_{2}$ in 3} & 3093.0 & 1185.0 & 5.68 & 2.51 & $\mathrm{CH}_{2}$ in 7 & 3098.0 & 1182.9 & 3.09 & -0.51 \\
\hline & 3089.1 & 1186.6 & -6.68 & -2.44 & & 3077.4 & 1191.9 & -4.64 & 0.50 \\
\hline \multirow[t]{2}{*}{$\mathrm{CH}_{2}$ in 5} & 3085.7 & 1188.0 & -4.12 & 0.44 & $\mathrm{CH}_{2}$ in 5 & 3114.1 & 1176.1 & 2.36 & 0.47 \\
\hline & 3093.9 & 1184.6 & -5.62 & -0.26 & & 3077.9 & 1191.4 & 6.12 & -0.28 \\
\hline \multirow[t]{2}{*}{$\mathrm{CH}_{2}$ in 6} & 3086.5 & 1187.7 & -1.42 & 0.68 & $\mathrm{CH}_{2}$ in 6 & 3084.2 & 1188.7 & -3.63 & 0.70 \\
\hline & 3082.1 & 1189.6 & 4.18 & -0.91 & & 3073.1 & 1193.4 & -3.66 & -0.93 \\
\hline \multirow{3}{*}{$\mathrm{CH}_{3}$ in 1} & 3090.3 & 1186.1 & -3.06 & 0.15 & $\mathrm{CH}_{3}$ in 1 & 3087.2 & 1187.4 & -0.19 & 0.15 \\
\hline & 3098.1 & 1182.8 & -1.38 & -0.12 & & 3095.5 & 1183.9 & -4.10 & -1.21 \\
\hline & 3104.6 & 1180.1 & 3.43 & 1.54 & & 3104.0 & 1180.4 & 4.02 & 1.51 \\
\hline \multirow[t]{3}{*}{$\mathrm{CH}_{3}$ in $7 \mathrm{a}$} & 3080.7 & 1190.2 & -5.58 & -0.62 & $\mathrm{CH}_{3}$ in $3 \mathrm{a}$ & 3108.7 & 1178.4 & 7.18 & 0.78 \\
\hline & 3089.9 & 1186.3 & 6.20 & 0.96 & & 3113.7 & 1176.3 & 4.63 & -0.64 \\
\hline & 3111.6 & 1177.2 & 0.66 & 0.04 & & 3083.0 & 1189.2 & -6.11 & 0.37 \\
\hline \multirow{3}{*}{$\mathrm{CH}_{3}$ in $7 \mathrm{~b}$} & 3129.1 & 1169.9 & 0.93 & 0.24 & $\mathrm{CH}_{3}$ in $3 \mathrm{~b}$ & 3115.5 & 1177.2 & 0.63 & 0.45 \\
\hline & 3088.1 & 1187.0 & -5.59 & -0.03 & & 3108.1 & 1178.6 & -7.87 & -0.11 \\
\hline & 3077.8 & 1191.4 & 0.95 & -0.28 & & 3083.8 & 1188.8 & 1.91 & -0.61 \\
\hline
\end{tabular}

${ }^{a}$ Assignment (1st columns), ab initio calculated frequencies for the fundamental ( $\Delta v=1,2$ nd columns), calculated wavelengths for the 2nd overtone $\left(\Delta v=3\right.$, 3rd columns) as proposed in the text. Calculated rotational strengths out of GAUSSIAN for $\Delta v=1$ in $10^{-44} \mathrm{esu}^{2} \mathrm{~cm}^{2}$ units $(4$ th columns) and based on the classical model presented in the text (5th columns) in $10^{-47} \mathrm{esu}^{2} \mathrm{~cm}^{2}$ units.

nicely in accord with the local mode hypothesis as described, e.g., in refs 13 and 14 . We have evaluated the overtone frequencies at $\Delta v_{\mathrm{s}}=3$ from eq 1 , using the various values of $\omega_{\mathrm{s}}{ }^{0}$ in Tables $2-4$ and $\chi_{\mathrm{s}}=70 \mathrm{~cm}^{-1}$ for all $\mathrm{CH}$ local modes. Such value for $\chi_{\mathrm{s}}$ is slightly higher than the experimental value for $\chi_{\mathrm{s}}$ (Table 1), to substitute the effect of scaling factors usually applied to $\omega_{\mathrm{s}}$. The corresponding calculated wavelength values at $\Delta v_{\mathrm{s}}=3$ are given in column 3 of Tables $2-4$. The same procedure would be applicable to $\Delta v_{\mathrm{s}}=4$.

As experimentally observed, a broad distribution in wavelength is predicted for $(3 R)-(+)$-methylcyclopentanone (Table $2)$ and $(3 R)-(+)$-methylcyclohexanone (Table 3$)$; a very narrow one is instead predicted for fenchone and camphor (Table 4).

(II) Dipole Strengths and Rotational Strengths. We propose that the relative values of dipole strengths and rotational strengths at $\Delta v_{\mathrm{s}}=3$ are the same as the relative values of dipole strengths and rotational strengths calculated at $\Delta v_{\mathrm{s}}=1$ by GAUSSIAN for the $16 d_{15}$-isotopomers of fenchone and camphor (or the $12 d_{11}$ ones or $10 d_{9}$ ones for (3R)-(+)methylcyclohexanone and $(3 R)-(+)$-methylcyclopentanone). This means ignoring electrical anharmonicity but assuming a contribution to intensity due to dynamical anharmonicity as if a $v_{\mathrm{s}}$, $\omega_{\mathrm{s}}{ }^{0}$, and $\chi_{\mathrm{s}}$ dependent scaling factor could be assumed connecting transition moments $0 \rightarrow v_{\mathrm{s}}$ with transition moments 0 $\rightarrow 1$. This scaling is not taken into account in the results presented below, since the purpose there is to reproduce the general shape of both the absorption and VCD spectra. Assuming dipole moment operators to linearly depend on $\mathrm{CH}$-stretching elongations $\left(l-l_{0}\right)$, it is appropriate to consider that for Morse local oscillators with mechanical frequency $\omega_{\mathrm{s}}{ }^{0}$ and anharmonicity $\chi_{\mathrm{s}}$, the transition moments of $\left(l-l_{0}\right)$ are given by ${ }^{39-41}$

$$
\begin{aligned}
& \left\langle 0\left|l-l_{0}\right| v_{\mathrm{s}}\right\rangle= \\
& \sqrt{\frac{v_{\mathrm{s}} !\left(2 K-2 v_{\mathrm{s}}-1\right)(2 K-1)}{(2 K-1)\left(2 K-v_{\mathrm{s}}\right)} \frac{(-1 / a)(-1)^{\mathrm{v}_{\mathrm{s}}}}{v_{\mathrm{s}}\left(2 K-v_{\mathrm{s}}-1\right)}} \cong \\
& (-1)^{\mathrm{v}_{\mathrm{s}}}(1 / a) \frac{1}{v_{\mathrm{s}}} \sqrt{\frac{v_{\mathrm{s}} !}{(2 \mathrm{~K})^{\mathrm{v}_{\mathrm{s}}}}}
\end{aligned}
$$

The first equation is valid for any $v_{\mathrm{s}}$; the latter one is valid for low values of $v_{\mathrm{s}}$. On this basis, the intensity scaling factor from the $0 \rightarrow 1$ transition to the $0 \rightarrow v_{\mathrm{s}}$ transition is the ratio of the squares of the transition moments of $\left(l-l_{0}\right)$, namely

$$
\left|\frac{\left\langle 0\left|l-l_{0}\right| v_{\mathrm{s}}\right\rangle}{\left\langle 0\left|l-l_{0}\right| 1\right\rangle}\right|^{2} \cong \frac{1}{v_{\mathrm{s}}^{2}}\left(\frac{v_{\mathrm{s}} !}{(2 K)^{\mathrm{v}_{\mathrm{s}}-1}}\right)
$$

where $\mathrm{K}=\omega_{\mathrm{s}}{ }^{0} / 2 \chi_{\mathrm{s}}$ and $a$ is the characteristic Morse potential parameter, related to $\chi_{\mathrm{s}} \cdot{ }^{17,18}$ For $v_{\mathrm{s}}=3$ such scaling factor is approximately 0.00035 for all VCD rotational strengths of Tables 2-4, columns 4 and 9 . This scaling factor would take these values calculated by GAUSSIAN for $\Delta v_{\mathrm{s}}=1$ to the order of $10^{-47} \mathrm{esu}^{2} \mathrm{~cm}^{2}$, which is what is expected. To facilitate comparison with experiment, we report in Figure 5 the four experimental absorption and VCD spectra at $\Delta v_{\mathrm{s}}=3$ for the four molecules under study. We compare them with calculated absorption and VCD spectra in Figure 6, obtained as follows. To each calculated VCD feature of Tables 2-4 plus the corresponding absorption features (and for the axial conformations of $\mathrm{CH}_{3}$ in the case of $(3 R)-(+)$-methylcyclopentanone and $(3 R)-(+)$-methylcyclohexanone), we prescribe a Lorentzian profile with the $\gamma$ parameter (see for example ref 5) equal to 10 $\mathrm{nm}$ and with areas equal to the calculated rotational strengths and dipole strengths, respectively. As a result of the energy difference in the two conformations for $(3 R)-(+)$-methylcyclopentanone and $(3 R)-(+)$-methylcyclohexanone, we have calculated the normalized Boltzmann coefficients at room temperature to be $89 \%$ for the "equatorial" and $11 \%$ for the "axial" conformer in $(3 R)-(+)$-methylcyclopentanone and $93 \%$ for the "equatorial" and $7 \%$ for the "axial" conformer in $(3 R)-(+)-$ methylcyclohexanone. The results may be considered quite encouraging as far as the absorption spectra of the four molecules are concerned and not too bad as far as the VCD spectra are concerned, except for $(1 S)-(+)$-fenchone. Indeed two facts are accounted for: we predict and observe a narrow absorption band for both camphor and fenchone, and we predict and observe a broad absorption band for both $(3 R)-(+)-$ methylcyclopentanone and $(3 R)-(+)$-methylcyclohexanone. Moreover the experimental VCD spectrum of $(1 S)-(-)$-camphor is predicted in the correct order, although the relative intensities are incorrect, for the two opposite components of the bisignate couplet observed in correspondence to the major absorption peak. The $(1 S)-(+)$-fenchone VCD spectrum is incorrectly accounted for. The VCD spectra of $(3 R)-(+)$-methylcyclopen- 

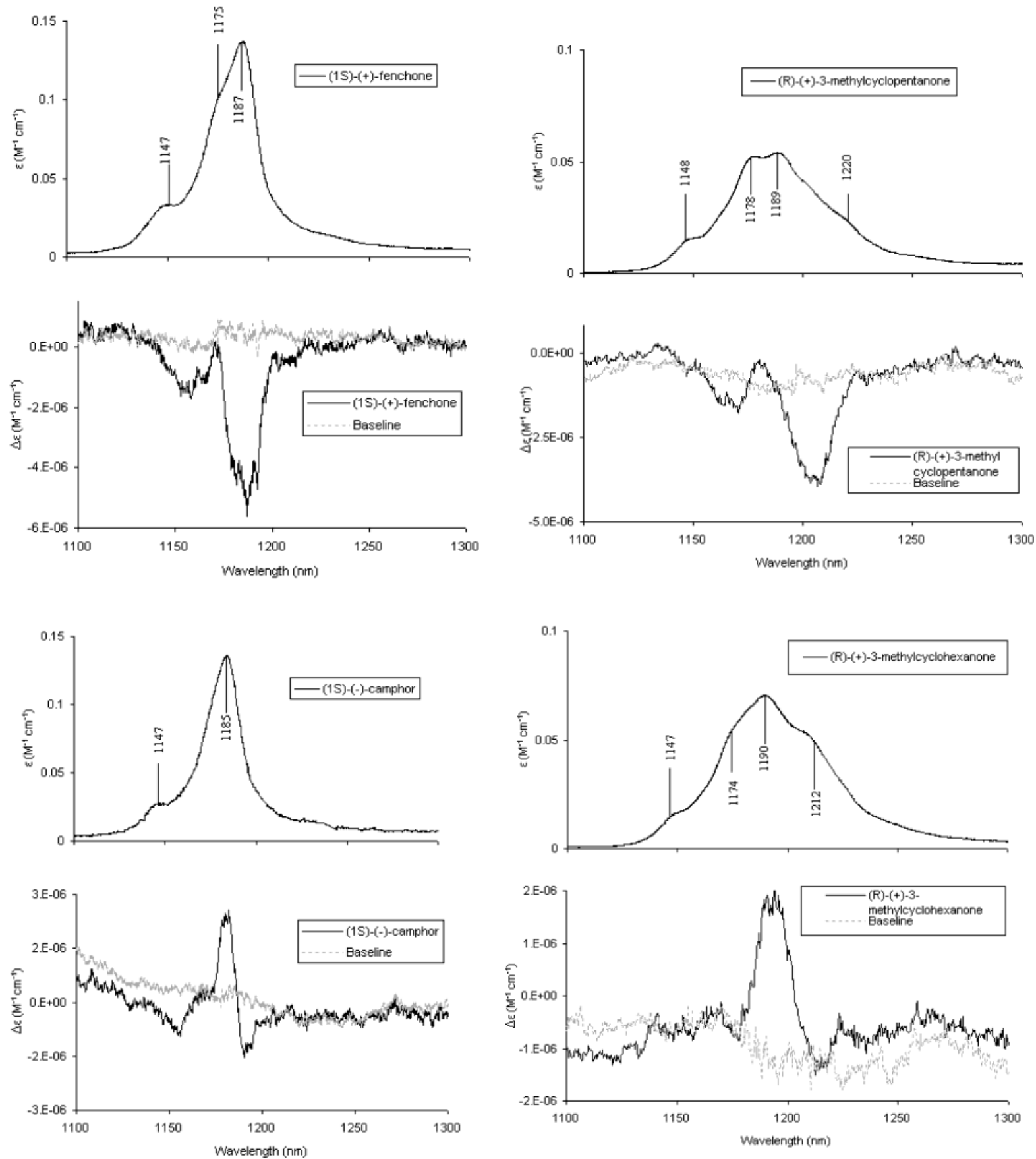

Figure 5. Experimental NIR absorption and VCD spectra of $(1 S)-(+)$-fenchone (top left), (1S)-(-)-camphor (bottom left), (3R)-(+)methylcyclopentanone (top right), and $(3 R)-(+)$-methylcyclohexanone (bottom right) in the range $1300-1100 \mathrm{~nm}\left(\Delta v_{\mathrm{s}}=3, \Delta v_{\mathrm{b}}=0\right)$. The data for the latter two molecules are repeated from ref 14. The VCD baselines in the figure are the VCD average spectra for fenchone and camphor as defined in Figures 1 and 2 and are the VCD spectra of the racemic compounds for $(3 R)-(+)$-methylcyclopentanone and $(3 R)-(+)$-methylcyclohexanone.

tanone and $(3 R)-(+)$-methylcyclohexanone are interpreted on the basis of the present calculations as both composed by two series of closely lying bands: the first one is overall negative at higher wavelength and the second one is overall positive at shorter wavelength. For some reason the negative one is weak for $(3 R)-(+)$-methylcyclohexanone and the positive one is weak for $(3 R)-(+)$-methylcyclopentanone. Indeed the experiment indicates just the presence of a strong negative VCD band for $(3 R)-(+)$-methylcyclopentanone and of a strong positive one for $(3 R)-(+)$-methylcyclohexanone; however, the two bands occur at different wavelengths, as predicted, and some minor features of opposite sign are also observed. As one may notice from Tables 2 and 3 though, the assignment of the negative and positive VCD feature is different for $(3 R)-(+)$-methyl- cyclopentanone and $(3 R)-(+)$-methylcyclohexanone, with the $\mathrm{CH}_{3}$ group in the equatorial position. In the former case the two bands arise mainly from $\mathrm{CH}$-stretchings in the $\alpha$-position, the higher wavelength one corresponding to pseudoaxial $\mathrm{CHs}$ and the lower wavelength one to pseudoequatorial ones. Such clear separation is not observed for $(3 R)-(+)$-methylcyclohexanone.

As stated above, our approach is merely heuristic and as such we ask the model to be judged and used on the basis of the results, which we consider satisfactory here. However, a justification of the validity of the present protocol lies on two facts, which constitute the basic hypotheses of the model:

(i) One does not need to calculate frequencies, dipole strengths, and rotational strengths for combination bands, the 

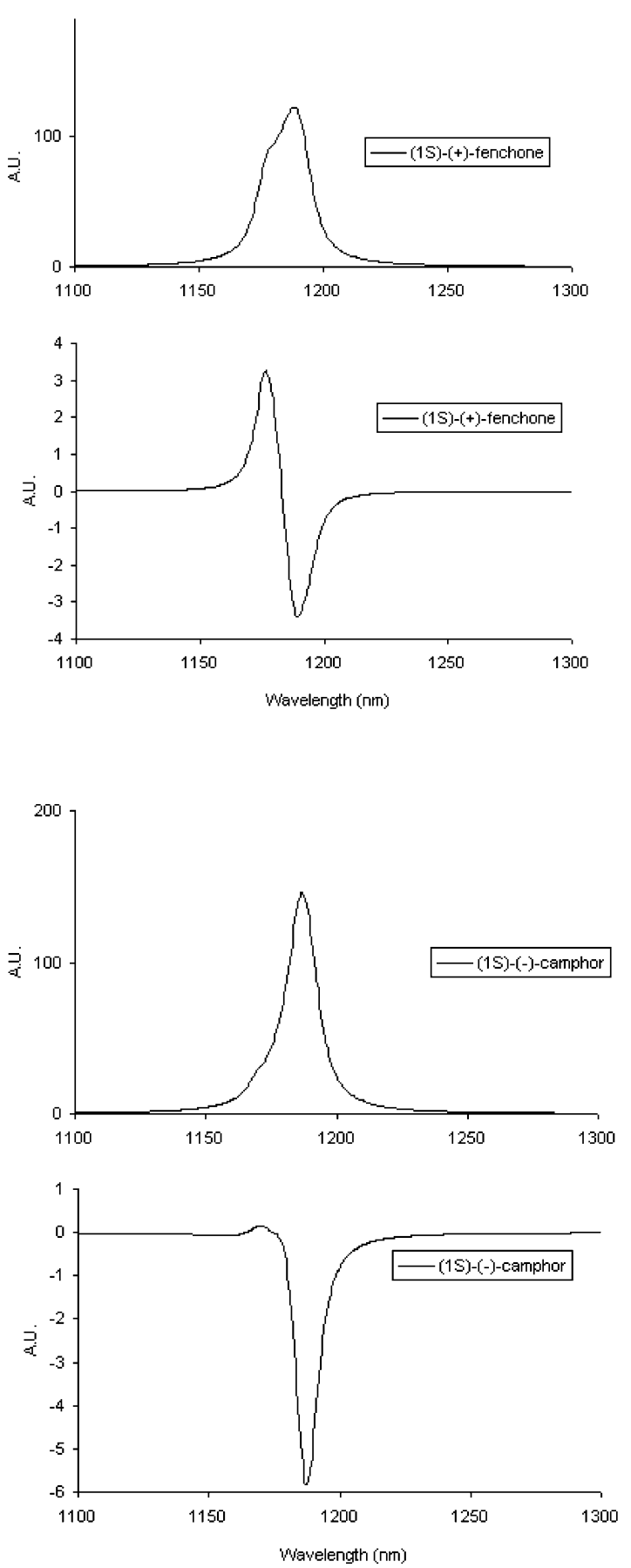
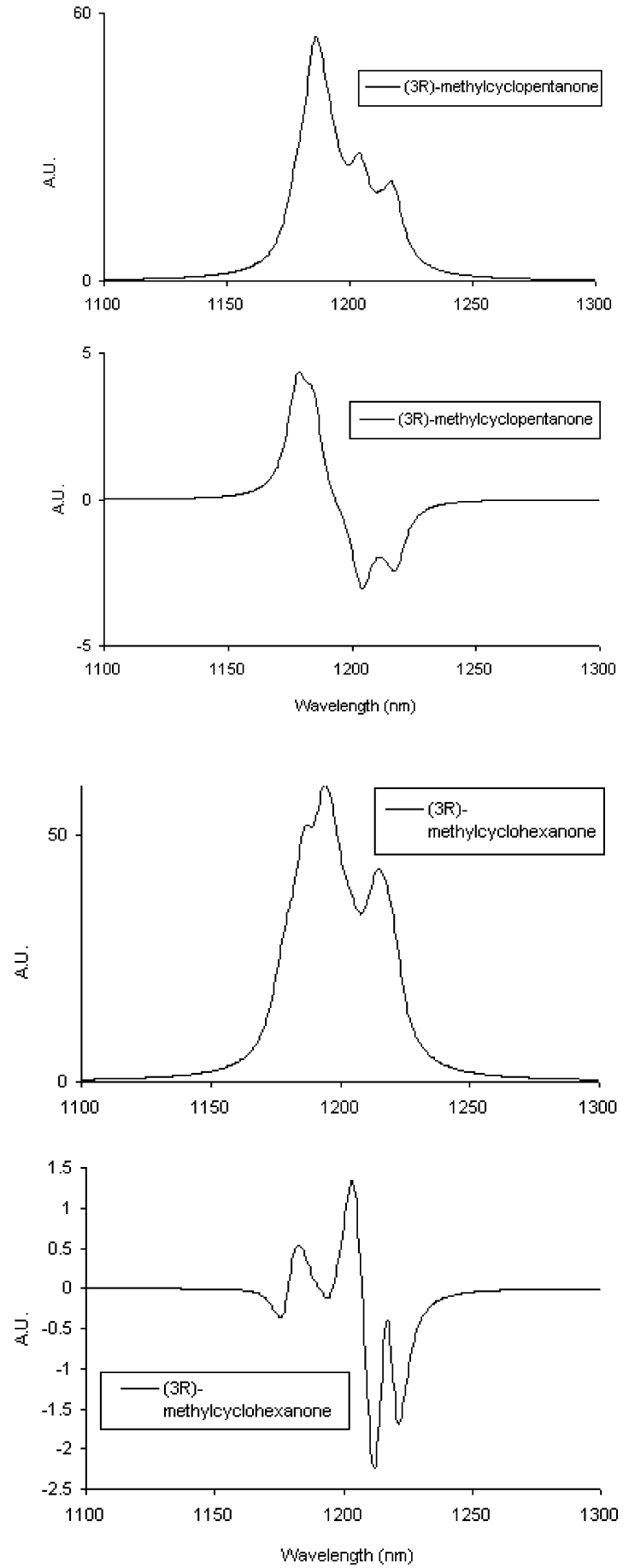

Figure 6. Calculated NIR absorption and VCD spectra of $(1 S)-(+)$-fenchone (top left), $(1 S)-(-)$-camphor (bottom left), $(3 R)-(+)-$ methylcyclopentanone (top right), and (3R)-(+)-methylcyclohexanone (bottom right) in the range 1300-1100 nm $\left(\Delta v_{\mathrm{s}}=3, \Delta v_{\mathrm{b}}=0\right)$. Calculations are based solely on the results of GAUSSIAN as described in the text. Lorentzian profiles assigned to each vibrational local mode transition with the appropriate dipole and rotational strength $(\gamma$ parameter $=10 \mathrm{~nm})$. For the latter two molecules the thermal averages for the conformers with the $\mathrm{CH}_{3}$ group in the equatorial and axial positions have been taken at room temperature (see text). Notice that the ordinate axes in both calculated absorption and VCD spectra are arbitrary.

number of which increases with the power of the overtone order (at $\Delta v_{\mathrm{s}}=3$ for fenchone and camphor it is 800 ). On the basis of the local mode hypothesis, ${ }^{17,18}$ the latter transitions have negligible probability to occur. One thus needs only to calculate dipole strengths and rotational strengths for the 16 local mode overtone transitions (for fenchone and camphor, and 12 and 10 for the other two molecules under examination).

(ii) To have nonzero dipole and rotational strengths for overtone transitions, one needs to have mechanical anharmo- nicity or electrical anharmonicity or both. To transfer, as we have done in step II of the present approach, the dipole strengths and rotational strengths from $\Delta v_{\mathrm{s}}=1$ to $\Delta v_{\mathrm{s}}=3$ is tantamount to complying with the hypothesis of electrical harmonicity and thus to exploiting the polar tensors and axial tensors ${ }^{42}$ calculated in the GAUSSIAN package. In this approach we effectively attribute to the sole mechanical anharmonicity, generating anharmonic wave functions, the possibility of observing absorption and VCD signals at the overtones' orders. In this hypothesis 

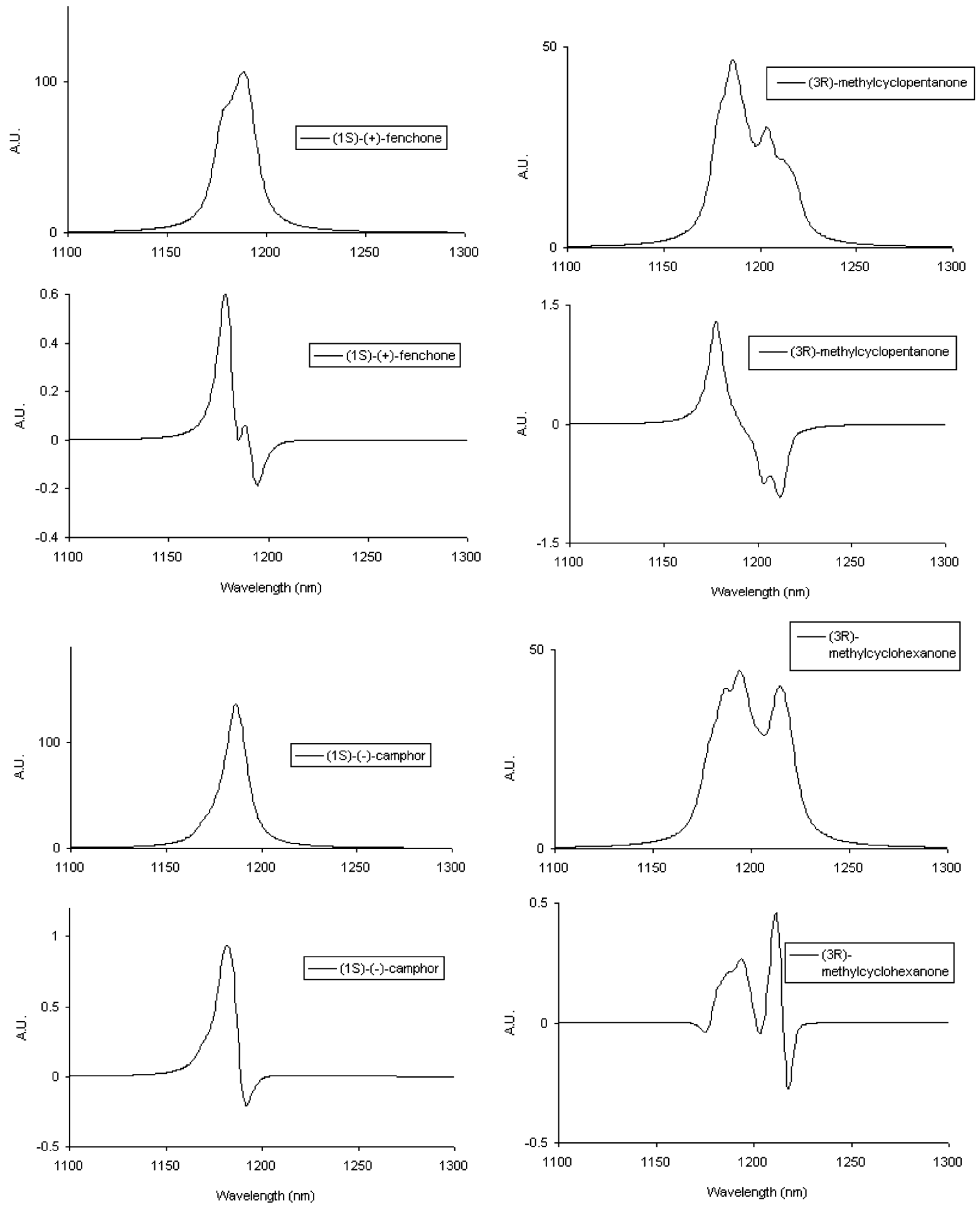

Figure 7. Calculated NIR absorption and VCD spectra of (1S)-(+)-fenchone (top left), (1S)-(-)-camphor (bottom left), $(3 R)-(+)$-methylcyclopentanone (top right), and $(3 R)-(+)$-methylcyclohexanone (bottom right) in the range $1300-1100 \mathrm{~nm}\left(\Delta v_{\mathrm{s}}=3, \Delta v_{\mathrm{b}}=0\right)$. Calculations are based on the results of GAUSSIAN for wavelengths and on the results of the polarizability model described in the text for the evaluation of dipole and rotational strengths. Lorentzian profiles assigned to each vibrational local mode transition with the appropriate dipole and rotational strength $(\gamma$ parameter $=$ $10 \mathrm{~nm}$ ). For the latter two molecules the thermal averages for the conformers with the $\mathrm{CH}_{3}$ group in the equatorial and axial positions have been taken at room temperature (see text). Notice that the ordinate axes in both calculated absorption and VCD spectra are arbitrary.

the dipole and rotational strengths for overtone transitions may be calculated from the corresponding quantities of the fundamental transition multiplied by the scaling factor given in eq $4^{\prime}$. In the case of almost equivalent oscillators, the scaling factor due to the mechanical part is almost identical for all oscillators and any difference has been ignored here.

(b2) Approach Based on ab Initio plus Classical Calculations. By this second approach we calculate the frequencies for just the local mode transitions exactly as done in paragraph b1. Instead, we calculate dipole and rotational strengths for the same transitions as follows. For each local mode associated with the $j$ th $\mathrm{CH}$-stretching $l_{j}$ in the $v_{j}$ th vibrational local mode overtone, we assume that the transition moment for the electric dipole moment is known: $\mu_{v j}=\left\langle 0|\mu| v_{j}\right\rangle$ (e.g., from experiments on overtones such as in ref 41). Following loosely the approach of ref 22 , we can calculate the instantaneous electric field $\mathbf{E}_{v j}$ 
generated by $\mu_{v j}$ at a given point $P$ with oriented distance $\mathbf{r}$ from the point dipole $\mu_{v j}$ by the equation

$$
\mathbf{E}_{v j}(\mathbf{r})=\left|\mu_{v j}\right|\left[3 \mathbf{r}\left(\mathbf{r} . \mathbf{e}_{j}\right)-\mathbf{e}_{j} r^{2}\right] / r^{5}
$$

Here $\mathbf{e}_{j}$ is the unit vector along the $j$ th $\mathrm{CH}$ bond and $r$ is the absolute value of $\mathbf{r}$. If at $\mathbf{r}$ there is a polarizable group with a polarizability tensor $((\alpha))$, an induced electric dipole moment is generated from the electric field of eq 5; we indicate it by $\mu_{v j}^{\mathrm{I}}$ and calculate it as

$$
\mu_{v j}^{\mathrm{I}}(\mathbf{r})=((\alpha)) \mathbf{E}_{v j}(\mathbf{r})
$$

Following ref 22, on the basis of the fundamental paper by Condon, ${ }^{43}$ we can calculate a subsequent magnetic dipole moment being generated as

$$
\left\langle v_{j}|\mathbf{m}| 0\right\rangle=\mathrm{i} \omega_{v j} \mathbf{r} \times \mu_{v j}{ }^{\mathrm{I}}
$$

where $\mathrm{i}$ is the imaginary unit and $\omega_{v j}$ is the frequency associated with the transition $|0\rangle \rightarrow\left|v_{j}\right\rangle$. The associated rotational strength $\mathscr{R}_{v j}$ is then calculated through the Rosenfeld formula and using eq 7 , i.e.

$$
\mathscr{R}_{v j}=\operatorname{Im}\left\langle 0|\mu| v_{j}\right\rangle \cdot\left\langle v_{j}|\mathbf{m}| 0\right\rangle
$$

At this point one can draw two conclusions: (a) eq 8 is originindependent since, due to eqs 7,6 , and 5, it depends on the relative distance $\mathbf{r}$ between inducing moment, where the actual vibration takes place, and the polarizable group. (b) the sign of $\mathscr{R}_{v j}$ is independent of the transition order and depends just on "static" properties such as geometry $\left(\mathbf{r}\right.$ and $\mathbf{e}_{j}$ ) or the polarizability $((\alpha))$. The latter property is important and may explain the observation that the sign of the observed rotational strengths is independent of $\Delta v_{\mathrm{s}} \cdot{ }^{13,14}$ In particular we notice that if the transition moments $\mu_{v j}$ changed from overtone to overtone, even reversing direction, this would still be true. Of course the prediction of the same qualitative VCD spectrum for all $\Delta v_{\mathrm{s}}$ is also contained in protocol b1)

We set $\left|\mu_{v j}\right|=0.353 \times 10^{-3}$ e $\AA$, as taken from Table 4 of ref 41 for $\Delta v_{\mathrm{s}}=3$ in cyclohexane $\mathrm{CHs}$ for all $j$, i.e., for all the $\mathrm{CH}$ bonds in $(1 S)-(+)$-fenchone, $(1 S)-(-)$-camphor, $(3 R)-(+)$ methylcyclohexanone, and $(3 R)-(+)$-methylcyclopentanone; for all four molecules we take a common polarizability tensor of the $\mathrm{C}=\mathrm{O}$ bond located at the center of the $\mathrm{C}=\mathrm{O}$ bond as

$$
((\alpha))\left[\AA^{3}\right]=\left[\begin{array}{lll}
2.0 & 0 & 0 \\
0 & 2.0 & 0 \\
0 & 0 & 2.3
\end{array}\right]
$$

The tensor in eq 9 is given in the principal axes of the group polarizability, where the $z$ axis coincides with the $\mathrm{C}=\mathrm{O}$ bond, the $x$ axis is perpendicular to the $\mathrm{CCC}=\mathrm{O}$ plane, and the $y$ axis lies in the $\mathrm{CCC}=\mathrm{O}$ plane and is perpendicular to $\mathrm{C}=\mathrm{O}$. These values are not too far from those of formaldehyde as reported by Applequist et al.; ${ }^{23}$ they are slightly lower than in formaldehyde in order to subtract the contribution of formaldehyde hydrogens and to account for just the effect of the $\mathrm{C}=\mathrm{O}$ bond. The geometries of all of the molecules, which are crucial for the calculations implied by eqs 4 and 6 , are for the most probable conformations calculated by GAUSSIAN $98^{19}$ (with the choice B3LYP/6-31G** for the functional/basis set).

The results are reported in Tables 2, 3, and 4 and in Figure 7 , where again the graphical results for $(3 R)-(+)$-methylcyclopentanone and $(3 R)-(+)$-methylcyclohexanone are the thermal
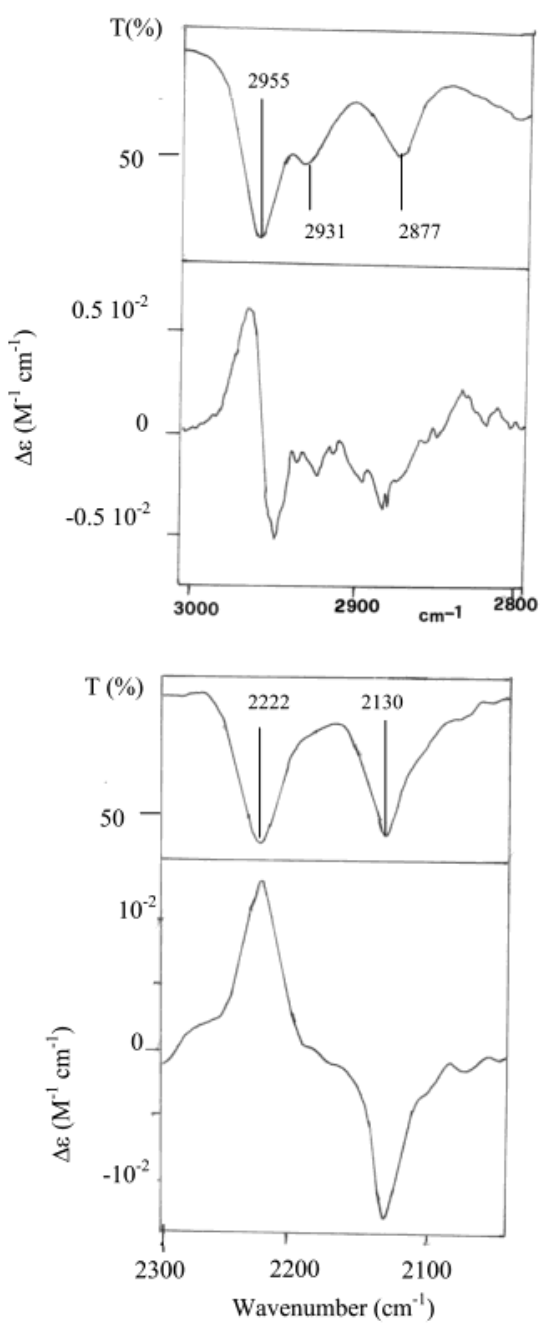

Figure 8. Experimental infrared absorption and circular dichroism spectra in the fundamental range of $\mathrm{CH}$-stretchings (top) and $\mathrm{CD}$ stretchings for 2,2,5,5- $d_{4^{-}}(3 R)-(+)$-methylcyclopentanone. Data taken at the University of Minnesota in 1982 with a dispersive apparatus. $\mathrm{CaF}_{2}$ cell with 0.06-mm path length used; $0.05 \mathrm{M}$ solution in $\mathrm{CCl}_{4}$.

averages for the equatorial and axial conformers for the $\mathrm{CH}_{3}$ group, whereas in Table 3 we just report the results for the most populated conformer. The results are of the same general quality as those reported with protocol b1. Still, with $(1 S)-(+)$-fenchone we predict a bisignate VCD feature, which the experiment does not bear. However the general aspect of the VCD and absorption spectra of $(1 S)-(-)$-camphor, $(3 R)-(+)$-methylcyclopentanone, and $(3 R)-(+)$-methylcyclohexanone is consonant with experiment and slightly better than what calculated with protocol b1. For fenchone still the general aspect of the absorption spectrum is correctly predicted by the model.

\section{Conclusions}

The study of the absorption and VCD spectra for the $\mathrm{CH}$ stretching fundamental, for the first three $\mathrm{CH}$-stretching overtone regions, and for the first three $\mathrm{CH}$-stretching/ $\mathrm{HCH}$-bending combination regions for camphor and fenchone has allowed us to clarify the role of $\mathrm{CH}$-stretchings in the generation of vibrational optical activity in the case of cyclic ketones. Second but also important, we were able to come up with a first qualitative interpretation of the role of $\mathrm{CH}$-stretchings in $\mathrm{CH}$ stretchings/HCH-bendings combinations regions. A correlative study of the data has allowed us to explain the importance of the cyclopentanone ring in the generation of vibrational optical 

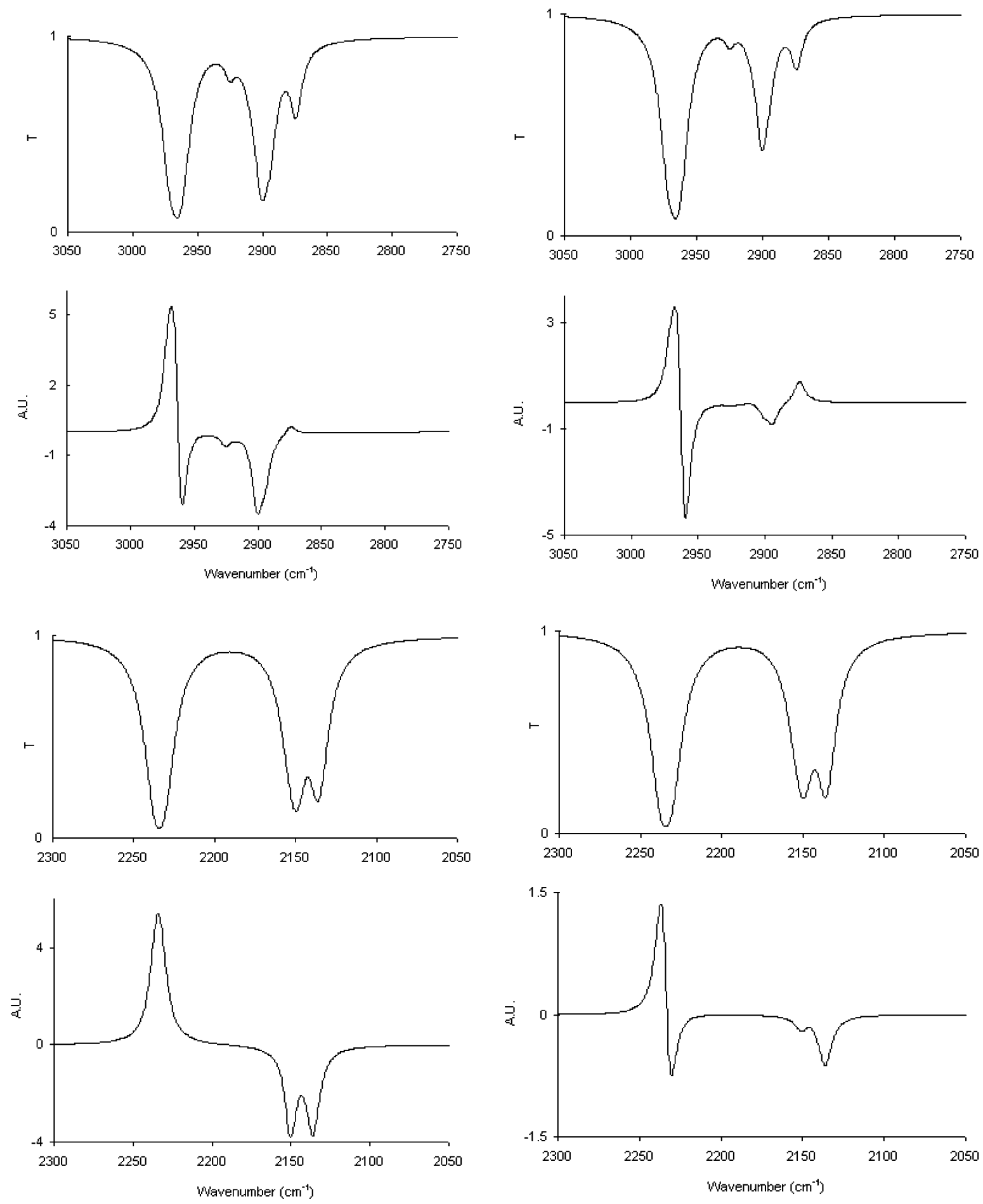

Figure 9. Calculated infrared absorption and vibrational circular dichroism spectra in the fundamental range of $\mathrm{CH}$-stretchings (top) and $\mathrm{CD}$ stretchings for 2,2,5,5- $d_{4^{-}}(3 R)-(+)$-methylcyclopentanone (bottom), according to the two approaches presented here. On the left we report the results obtained with GAUSSIAN for the frequencies, dipole strengths, and rotational strengths. On the right we report the results obtained with GAUSSIAN for frequencies and with the classical model discussed in the text for dipole strengths and rotational strengths. In all cases Lorentzian profiles were assigned to all transitions with $\gamma=10 \mathrm{~cm}^{-1}$. The thermal average for the conformers with the $\mathrm{CH}_{3}$ group in the equatorial and axial positions has been taken at room temperature (see text). Notice that the ordinate axes in both calculated transmittance and VCD spectra are arbitrary.

activity in the near-infrared for these molecules. In particular we have pointed out that the three $\mathrm{CH}$-stretchings in the cyclopentanone ring of camphor and fenchone could account for the observed VCD.

In the final part of the paper we report on two types of calculations, which are both based on the calculations of harmonic mechanical frequencies by use of an ab initio package for $\mathrm{H}_{1}$-isotopomers, the rest of the hydrogen atoms being substituted by deuterium atoms. Complementing these calculations with the use of the Birge-Sponer law with an ad hoc, but in any case reasonable, choice for the value of the anharmonicity constant, we find a quite acceptable interpretation of the bandwidth and shape of the overtone absorption and VCD spectra. The two approaches differ in the calculation of dipole and rotational strengths: in the first case we transfer the results of the ab initio calculations for $\Delta v_{\mathrm{s}}=1$ to the region $\Delta v_{\mathrm{s}}=3$ 
and further; in the second one we employ a model that allows us to evaluate the contribution of the polarizability of the $\mathrm{C}=\mathrm{O}$ to dipole and rotational strengths.

The two approaches provide qualitatively similar results and this makes us think that the mechanism for the generation of the magnetic dipole moment due to local stretching vibrations is largely due to polarization of the electrons in the $\mathrm{C}=\mathrm{O}$ bond. The polarizability effect is particularly important for $\mathrm{CH}$ close to the $\mathrm{C}=\mathrm{O}$ bond (see eq 5), and this may explain why the VCD spectra of $(1 S)-(+)$-fenchone are not correctly interpreted, no $\mathrm{CH}$ bond being close to $\mathrm{C}=\mathrm{O}$. Also, this may give some credit to empirical interpretations of the VCD spectra presented at the beginning of the paper, namely that the only $\mathrm{CHs}$ being important for VCD are those of the cyclopentanone ring.

The question now arises whether these approaches may be extended to either the fundamental $\mathrm{CH}$-stretching region or to other $\mathrm{CH}$-stretching overtone regions. We think that this is viable for $\Delta v_{\mathrm{s}}=4$ and over, where the local mode scheme works. However, we defer this to later studies, whereby the evaluation of $\chi_{\mathrm{s}}$ be also made via ab initio methods. Indeed the extension to $\Delta v_{\mathrm{s}}=4$ implies that VCD and absorption spectra be identical in shape to $\Delta v_{\mathrm{s}}=3$, and this is what is actually observed (see Figures 1 and 2 and refs 13 and 14). For $\Delta v_{\mathrm{s}}=1$ (fundamental region) normal modes are definitely present; ${ }^{17,18,27,31}$ however, unless strong anharmonic effects, such as Fermi resonance, are present, the straight calculations from GAUSSIAN on the "undeuterated" molecule provides acceptable results; the polarizability approach instead should be complemented with addition of coupled dipole moments contributions. We did so for $\alpha, \alpha, \alpha^{\prime}, \alpha^{\prime}-d_{4}-(3 R)-(+)$-methylcyclopentanone, for which we report in Figure 8 the experimental absorption and VCD spectra in the $\mathrm{CH}$-stretching and $\mathrm{CD}$-stretching regions. In Figure 9 we report the results of the two types of calculations, where the frequencies were evaluated still by the use of eq 1 with $\chi_{\mathrm{s}}=$ $35 \mathrm{~cm}^{-1}$ for all CD-stretching modes and $\chi_{\mathrm{s}}=70 \mathrm{~cm}^{-1}$ for all $\mathrm{CH}$-stretching modes. This is consistent with what was done for $\Delta v_{\mathrm{s}}=3$, since although harmonic frequencies are inversely proportional to the square root of the mass, anharmonicities are inversely proportional to the mass. ${ }^{17,18,20}$ Besides, in the second type of calculations, we set $\left|\mu_{v j}\right|=1.33 \times 10^{-2}$ e $\AA$, as taken from Table 4 of ref 41 for $v_{\mathrm{s}}=1$ in cyclohexane, to all $\mathrm{CH}$ (and CD) bonds of the molecule under study. We point out that in Figure 9 the thermal averages for the two most populated conformers for $(3 R)-(+)$-methylcyclopentanone were considered, with the appropriate Boltzmann coefficients calculated at room temperature. The results are quite good for both types of calculations: the bisignate feature at high wavenumbers for the second type of calculations is due to two closely lying features of opposite sign, the sum of which is positive, as of the experiment; the negative feature would not manifest itself if the $\gamma$ parameter of the Lorentzian profile were larger. We have verified that in the $\mathrm{CD}$-stretching region the dipole-dipole coupling contribution, which is the contribution most typical of normal modes, is not as important as the polarizability contribution. Instead, for the highest frequency bands of the $\mathrm{CH}$-stretching region, it works the other way around. Finally we notice that the interpretation of the $\Delta v_{\mathrm{s}}=2$ region is impossible at the moment, since therein the presence and relevance of combination bands has been amply demonstrated in the literature ${ }^{17,18,32}$ whereas here the combination modes are neglected.

Acknowledgment. Financial support from Italian Ministry of Education, University, and Research (MIUR) is gratefully acknowledged. We also thank Dr. Leo Laux of Lockheed
Corporation for making available to us the IR-VCD data of $\alpha, \alpha, \alpha^{\prime}, \alpha^{\prime}-d_{4}-(3 R)-(+)$-methylcyclopentanone; the data had been collected during his $\mathrm{Ph} . \mathrm{D}$. thesis work in 1982 at the University of Minnesota.

\section{References and Notes}

(1) Lightner, D. A.; Gurst, J. E. Organic Conformational Analysis and Stereochemistry from Circular Dichroism Spectroscopy; Wiley-VCH: 2000.

(2) Coulombeau, C.; Rassat, A. Bull. Soc. Chim. Fr. 1966, 3752-3762.

(3) Pulm, F.; Schramm, J.; Lagier, H.; Hormes, J. Enantiomer 1998 $3,315-322$.

(4) Pulm, F.; Schramm, J.; Hormes, J.; Grimme, S.; Peyerimhoff, S. D. Chem. Phys. 1997, 224, 143-155.

(5) Devlin, F. J.; Stephens, P. J.; Cheeseman, J. R.; Frisch, M. J. J. Phys. Chem. A 1997, 101, 6322-6333.

(6) Nafie, L. A. Advances in Applied FTIR Spectroscopy; MacKenzie, M. W., Ed. Wiley: New York, 1988; pp 67-104.

(7) Polavarapu, P. L.; Chen, G. C.; Weibel, S. Appl. Spectrosc. 1994, $48,1224-1234$

(8) Bour, P.; McCann, J.; Wieser, H. J. Phys. Chem. A 1998, 102, $102-110$.

(9) Stephens, P. J.; Devlin, F. J.; Cheeseman, J. R.; Frisch, M. J. J. Phys. Chem. A 2001, 105, 5356-5371.

(10) Mennucci, B.; Tomasi, J.; Cammi, R.; Cheesemann, J. R.; Frisch, M. J.; Devlin, F. J.; Gabriel, S.; Stephens, P. J. J. Phys. Chem. A 2002, 106, 6102-6113.

(11) Polavarapu, P. L. Chirality 2003, 14, 768-781.

(12) Giorgio, E.; Minichino, C.; Viglione, R. G.; Zanasi, R.; Rosini, C. J. Org. Chem. 2003, 68, 5186-5192.

(13) Abbate, S.; Longhi, G.; Boiadjiev, S.; Lightner, D. A.; Bertucci, C.; Salvadori, P. Enantiomer 1998, 5, 337-347.

(14) Castiglioni, E.; Lebon, F.; Longhi, G.; Abbate, S. Enantiomer 2002, $7,161-173$

(15) Keiderling, T. A.; Stephens, P. J. Chem. Phys. Lett. 1976, 41, 46-

(16) Nafie, L. A.; Cao, X.; Guo, C. Appl. Spectrosc. 2003, 57, 12451249. Also: Cao, X.; Guo, C.; Nafie, L. A. Note P36 presented at CD2003International Conference, Budapest, Hungary, September 2003. Also, private communication to one of the authors (S.A.).

(17) Henry, B. R. Acc. Chem. Res. 1987, 20, 429-435.

(18) Child, M. S.; Halonen, L. Adv. Chem. Phys. 1985, LVII, 1-58

(19) Frisch, M. J.; Trucks, G. W.; Schegek, H. B.; Scuseria, G. E.; Robb, M. A.; Cheesman, J. R.; Zakrzewski, V. G.; Montgomery, J. A., Jr.; Stratmenn, R. E.; Burant, J. C.; Dapprich, S.; Millam, J. M.; Daniels, A. D.; Kudin, K. N.; Strain, M. C.; Farkas, O.; Tomasi, J.; Barone, V.; Cossi, M.: Cammi, R.; Mennucci, B.; Pomelli, C.; Adamo, C.; Clifford, S.; Ocheterski, J.; Petersson G. A.; Ayala, PY.; Cui, Q.; Morokuma, K.; Malik, D. K.; Rabul, A. D.; Raghavachari, K.; Foresman, J. B.; Cioslowski, J.; Ortiz, J. V.; Stefanov, B. B.; Liu, G.; Liashenko, A.; Piskorz, P.; Komaromi, I.; Gomperts, R.; Martin, R. L.; Fox D. J.; Keith, T.; Al. Laham, M. A.; Peng C. Y.; Nanayakkara, A.; Gonzalez, C.; Challacombe, M.; Gill, P. M. W.; Johnson, B. G.; Chem, W.; Wong, M. W.; Andres, J. L.; Head-Gordon, M.; Replogle, E. S.; Pople, J. A. Gaussian 98, revision A.3; Gaussian Inc.: Pittsburgh, PA, 1998

(20) Herzberg, G., Infrared and Raman Spectra of Diatomic Molecules; Van Nostrand: New York, 1950

(21) Barnett, C. J.; Drake, A. F.; Kuroda, R.; Mason, S. F. Mol. Phys 1980, 41, 455-468.

(22) Abbate, S.; Havel, H. A.; Laux, L.; Pultz, V.; Moscowitz, A. J. Phys. Chem. 1988, 92, 3302-3311.

(23) Applequist, J.; Carl, J. R.; Fung, K.-K. J. Am. Chem. Soc. 1972, 94, 2952-2960.

(24) Applequist, J. J. Chem. Phys. 1973, 58, 4251-4259.

(25) DeVoe, H. J. Chem. Phys. 1965, 43, 3199-3208.

(26) Rosini, C.; Zandomeneghi, M. Gazz. Chim. Ital. 1981, 111, 493497.

(27) Devlin, F. J.; Stephens, P. J. J. Am. Chem. Soc. 1999, 121, 74137414.

(28) See for example: Pultz, V. M.; Abbate, S.; Laux, L.; Havel, H. A.; Overend, J.; Moscowitz, A.; Mosher, H. S. J. Phys. Chem. 1984, 88 , 505-507.

(29) Fuhrer, H.; Kartha, V. B.; Krueger, P. J.; Mantsch, H.; Jones, R. N. Chem. Rev. 1972, 72, 439-456.

(30) Laux, L. Ph.D. Thesis, Department of Chemistry, University of Minnesota, 1982

(31) Laux, L.; Pultz, V.; Abbate, S.; Havel, H. A.; Overend, J.; Moscowitz, A.; Lightner, D. A. J. Am. Chem. Soc. 1982, 104, 4276-4278. (32) Lespade, L.; Longhi, G.; Abbate, S. Chem. Phys. 1990, 142, 245259.

(33) Abbate, S.; Longhi, G.; Santina, C. Chirality 2000, 12, 180-190. 
(34) Henry, B. R.; Hung, I.; MacPhail, R. A.; Strauss, H. L. J. Am. Chem. Soc. 1980, 102, 515 .

(35) Ghisletti, D. Studio della Dinamica Molecolare mediante Spettrocopia nel Vicino-Infrarosso e Calcoli di Meccanica Classica; Tesi di Laurea in Fisica: Università di Milano, Italy, 1991.

(36) McKean, D. C. Chem. Soc. Rev. 1978, 7, 339-415.

(37) Stephens P. J.; Devlin F. J.; Chabalowski C. F.; Frisch M. J. J. Phys. Chem. 1994, 98, 11623. Stephens P. J.; Devlin F. J.; Ashwar, C. S.; Chabalowski, C. F.; Frisch, M. J. Faraday Discuss. 1994, 99, 103.

(38) Petersson, G. A.; Bennett, A.; Tensfeldt, T. G.; Al-Laham, M. A.; Shirley, W. A.; Mantzaris, J. J. Chem. Phys. 1988, 89, 2193
(39) Longhi G. Spettroscopie vibrazionali di glucosio e di molecole modello per glucosio; Tesi di Laurea in Fisica: Università di Milano, Italy, 1984.

(40) Gallas, J, A. C. Phys. Rev. 1980, 21, 1829. Fox, K. Phys. Lett. 1984, 104, 21.

(41) Zerbi, G.; Longhi, G.; Ricard, L.; Abbate, S. J. Chem. Phys. 1988, 88,6733 .

(42) Stephens, P. J. J. Phys. Chem. 1985, 89, 748-750. Also: Stephens, P. J. In Understanding Molecular Properties; Avery, J., Dahl, J. P., Hansen, A. E., Eds.; D. Reidel: Dordrecht, 1987; p 333.

(43) Condon, U. Rev. Mod. Phys. 1932, 9, 432-457. 